DESY-09-046

March 2009

\title{
Measurement of the longitudinal proton structure function at HERA
}

\author{
ZEUS Collaboration
}

\begin{abstract}
The reduced cross sections for ep deep inelastic scattering have been measured with the ZEUS detector at HERA at three different centre-of-mass energies, 318, 251 and $225 \mathrm{GeV}$. From the cross sections, measured double differentially in Bjorken $x$ and the virtuality, $Q^{2}$, the proton structure functions $F_{L}$ and $F_{2}$ have been extracted in the region $5 \times 10^{-4}<x<0.007$ and $20<Q^{2}<130 \mathrm{GeV}^{2}$.
\end{abstract}




\section{The ZEUS Collaboration}

S. Chekanov, M. Derrick, S. Magill, B. Musgrave, D. Nicholass ${ }^{1}$, J. Repond, R. Yoshida Argonne National Laboratory, Argonne, Illinois 60439-4815, USA ${ }^{n}$

M.C.K. Mattingly

Andrews University, Berrien Springs, Michigan 49104-0380, USA

P. Antonioli, G. Bari, L. Bellagamba, D. Boscherini, A. Bruni, G. Bruni, F. Cindolo, M. Corradi, G. Iacobucci, A. Margotti, R. Nania, A. Polini INFN Bologna, Bologna, Italy ${ }^{e}$

S. Antonelli, M. Basile, M. Bindi, L. Cifarelli, A. Contin, S. De Pasquale ${ }^{2}$, G. Sartorelli, A. Zichichi University and INFN Bologna, Bologna, Italy ${ }^{e}$

D. Bartsch, I. Brock, H. Hartmann, E. Hilger, H.-P. Jakob, M. Jüngst, A.E. Nuncio-Quiroz, E. Paul, U. Samson, V. Schönberg, R. Shehzadi, M. Wlasenko Physikalisches Institut der Universität Bonn, Bonn, Germany ${ }^{b}$

N.H. Brook, G.P. Heath, J.D. Morris

H.H. Wills Physics Laboratory, University of Bristol, Bristol, United Kingdom ${ }^{m}$

M. Kaur, P. Kaur ${ }^{3}$, I. Singh ${ }^{3}$

Panjab University, Department of Physics, Chandigarh, India

M. Capua, S. Fazio, A. Mastroberardino, M. Schioppa, G. Susinno, E. Tassi

Calabria University, Physics Department and INFN, Cosenza, Italy ${ }^{e}$

J.Y. Kim

Chonnam National University, Kwangju, South Korea

Z.A. Ibrahim, F. Mohamad Idris, B. Kamaluddin, W.A.T. Wan Abdullah

Jabatan Fizik, Universiti Malaya, 50603 Kuala Lumpur, Malaysia ${ }^{r}$

Y. Ning, Z. Ren, F. Sciulli

Nevis Laboratories, Columbia University, Irvington on Hudson, New York 10027, USA ${ }^{\circ}$

J. Chwastowski, A. Eskreys, J. Figiel, A. Galas, K. Olkiewicz, B. Pawlik, P. Stopa,

L. Zawiejski

The Henryk Niewodniczanski Institute of Nuclear Physics, Polish Academy of Sciences, Cracow, Poland ${ }^{i}$

L. Adamczyk, T. Bołd, I. Grabowska-Bołd, D. Kisielewska, J. Łukasik ${ }^{4}$, M. Przybycień, L. Suszycki

Faculty of Physics and Applied Computer Science, AGH-University of Science and Technology, Cracow, Poland ${ }^{p}$ 
A. Kotański ${ }^{5}$, W. Słomiński ${ }^{6}$

Department of Physics, Jagellonian University, Cracow, Poland

O. Behnke, J. Behr, U. Behrens, C. Blohm, K. Borras, D. Bot, R. Ciesielski, N. Coppola, S. Fang, A. Geiser, P. Göttlicher ${ }^{7}$, J. Grebenyuk, I. Gregor, T. Haas, W. Hain, A. Hüttmann, F. Januschek, B. Kahle, I.I. Katkov ${ }^{8}$, U. Klein ${ }^{9}$, U. Kötz, H. Kowalski, M. Lisovyi, E. Lobodzinska, B. Löhr, R. Mankel ${ }^{10}$, I.-A. Melzer-Pellmann, S. Miglioranzi ${ }^{11}$, A. Montanari, T. Namsoo, D. Notz, A. Parenti, P. Roloff, I. Rubinsky, U. Schneekloth, A. Spiridonov ${ }^{12}$, D. Szuba ${ }^{13}$, J. Szuba ${ }^{14}$, T. Theedt, J. Tomaszewska ${ }^{15}$, G. Wolf, K. Wrona, A.G. Yagües-Molina, C. Youngman, W. Zeuner ${ }^{10}$

Deutsches Elektronen-Synchrotron DESY, Hamburg, Germany

V. Drugakov, W. Lohmann, S. Schlenstedt

Deutsches Elektronen-Synchrotron DESY, Zeuthen, Germany

G. Barbagli, E. Gallo

INFN Florence, Florence, Italy ${ }^{e}$

P. G. Pelfer

University and INFN Florence, Florence, Italy $^{e}$

A. Bamberger, D. Dobur, F. Karstens, N.N. Vlasov ${ }^{16}$

Fakultät für Physik der Universität Freiburg i.Br., Freiburg i.Br., Germany ${ }^{b}$

P.J. Bussey, A.T. Doyle, M. Forrest, D.H. Saxon, I.O. Skillicorn

Department of Physics and Astronomy, University of Glasgow, Glasgow, United Kingdom ${ }^{m}$

I. Gialas ${ }^{17}$, K. Papageorgiu

Department of Engineering in Management and Finance, Univ. of Aegean, Greece

U. Holm, R. Klanner, E. Lohrmann, H. Perrey, P. Schleper, T. Schörner-Sadenius, J. Sztuk, H. Stadie, M. Turcato

Hamburg University, Institute of Exp. Physics, Hamburg, Germany ${ }^{b}$

C. Foudas, C. Fry, K.R. Long, A.D. Tapper

Imperial College London, High Energy Nuclear Physics Group, London, United Kingdom ${ }^{m}$

T. Matsumoto, K. Nagano, K. Tokushuku ${ }^{18}$, S. Yamada, Y. Yamazaki ${ }^{19}$

Institute of Particle and Nuclear Studies, KEK, Tsukuba, Japan ${ }^{f}$

A.N. Barakbaev, E.G. Boos, N.S. Pokrovskiy, B.O. Zhautykov

Institute of Physics and Technology of Ministry of Education and Science of Kazakhstan, Almaty, Kazakhstan 
V. Aushev ${ }^{20}$, O. Bachynska, M. Borodin, I. Kadenko, O. Kuprash, V. Libov, D. Lontkovskyi, I. Makarenko, Iu. Sorokin, A. Verbytskyi, O. Volynets, M. Zolko

Institute for Nuclear Research, National Academy of Sciences, and Kiev National University, Kiev, Ukraine

D. Son

Kyungpook National University, Center for High Energy Physics, Daegu, South Korea ${ }^{g}$

J. de Favereau, K. Piotrzkowski

Institut de Physique Nucléaire, Université Catholique de Louvain, Louvain-la-Neuve, Belgium ${ }^{q}$

F. Barreiro, C. Glasman, M. Jimenez, J. del Peso, E. Ron, J. Terrón, C. Uribe-Estrada Departamento de Física Teórica, Universidad Autónoma de Madrid, Madrid, Spain ${ }^{l}$

F. Corriveau, J. Schwartz, C. Zhou

Department of Physics, McGill University, Montréal, Québec, Canada H3A 2T8 ${ }^{a}$

T. Tsurugai

Meiji Gakuin University, Faculty of General Education, Yokohama, Japan ${ }^{f}$

A. Antonov, B.A. Dolgoshein, D. Gladkov, V. Sosnovtsev, A. Stifutkin, S. Suchkov Moscow Engineering Physics Institute, Moscow, Russia ${ }^{j}$

R.K. Dementiev, P.F. Ermolov ${ }^{\dagger}$, L.K. Gladilin, Yu.A. Golubkov, L.A. Khein, I.A. Korzhavina, V.A. Kuzmin, B.B. Levchenko ${ }^{21}$, O.Yu. Lukina, A.S. Proskuryakov, L.M. Shcheglova, D.S. Zotkin

Moscow State University, Institute of Nuclear Physics, Moscow, Russia ${ }^{k}$

I. Abt, A. Caldwell, D. Kollar, B. Reisert, W.B. Schmidke

Max-Planck-Institut für Physik, München, Germany

G. Grigorescu, A. Keramidas, E. Koffeman, P. Kooijman, A. Pellegrino, H. Tiecke, M. Vázquez ${ }^{11}$, L. Wiggers

NIKHEF and University of Amsterdam, Amsterdam, Netherlands ${ }^{h}$

N. Brümmer, B. Bylsma, L.S. Durkin, A. Lee, T.Y. Ling

Physics Department, Ohio State University, Columbus, Ohio 43210, USA ${ }^{n}$

P.D. Allfrey, M.A. Bell, A.M. Cooper-Sarkar, R.C.E. Devenish, J. Ferrando, B. Foster,

C. Gwenlan ${ }^{22}$, K. Horton ${ }^{23}$, K. Oliver, A. Robertson, R. Walczak

Department of Physics, University of Oxford, Oxford United Kingdom ${ }^{m}$

A. Bertolin, F. Dal Corso, S. Dusini, A. Longhin, L. Stanco

INFN Padova, Padova, Italy ${ }^{e}$

R. Brugnera, R. Carlin, A. Garfagnini, S. Limentani

Dipartimento di Fisica dell' Università and INFN, Padova, Italy ${ }^{e}$ 
B.Y. Oh, A. Raval, J.J. Whitmore ${ }^{24}$

Department of Physics, Pennsylvania State University, University Park, Pennsylvania $16802^{\circ}$

Y. Iga

Polytechnic University, Sagamihara, Japan ${ }^{f}$

G. D'Agostini, G. Marini, A. Nigro

Dipartimento di Fisica, Università 'La Sapienza' and INFN, Rome, Italy ${ }^{e}$

J.E. Cole ${ }^{25}$, J.C. Hart

Rutherford Appleton Laboratory, Chilton, Didcot, Oxon, United Kingdom ${ }^{m}$

H. Abramowicz ${ }^{26}$, R. Ingbir, S. Kananov, A. Levy, A. Stern

Raymond and Beverly Sackler Faculty of Exact Sciences, School of Physics, Tel Aviv

University,

Tel Aviv, Israel ${ }^{d}$

M. Kuze, J. Maeda

Department of Physics, Tokyo Institute of Technology, Tokyo, Japan ${ }^{f}$

R. Hori, S. Kagawa ${ }^{27}$, N. Okazaki, S. Shimizu, T. Tawara

Department of Physics, University of Tokyo, Tokyo, Japan ${ }^{f}$

R. Hamatsu, H. Kaji ${ }^{28}$, S. Kitamura ${ }^{29}$, O. Ota ${ }^{30}$, Y.D. Ri

Tokyo Metropolitan University, Department of Physics, Tokyo, Japan ${ }^{f}$

M. Costa, M.I. Ferrero, V. Monaco, R. Sacchi, V. Sola, A. Solano

Università di Torino and INFN, Torino, Italy ${ }^{e}$

M. Arneodo, M. Ruspa

Università del Piemonte Orientale, Novara, and INFN, Torino, Italy ${ }^{e}$

S. Fourletov ${ }^{31}$, J.F. Martin, T.P. Stewart

Department of Physics, University of Toronto, Toronto, Ontario, Canada M5S 1A7 a

S.K. Boutle ${ }^{17}$, J.M. Butterworth, T.W. Jones, J.H. Loizides, M. Wing ${ }^{32}$

Physics and Astronomy Department, University College London, London, United Kingdom ${ }^{m}$

B. Brzozowska, J. Ciborowski ${ }^{33}$, G. Grzelak, P. Kulinski, P. Łużniak ${ }^{34}$, J. Malka ${ }^{34}$, R.J. Nowak, J.M. Pawlak, W. Perlanski ${ }^{34}$, A.F. Żarnecki

Warsaw University, Institute of Experimental Physics, Warsaw, Poland

M. Adamus, P. Plucinski ${ }^{35}$

Institute for Nuclear Studies, Warsaw, Poland

Y. Eisenberg, D. Hochman, U. Karshon

Department of Particle Physics, Weizmann Institute, Rehovot, Israel ${ }^{c}$ 
E. Brownson, D.D. Reeder, A.A. Savin, W.H. Smith, H. Wolfe

Department of Physics, University of Wisconsin, Madison, Wisconsin 53706, USA ${ }^{n}$

S. Bhadra, C.D. Catterall, Y. Cui, G. Hartner, S. Menary, U. Noor, J. Standage, J. Whyte Department of Physics, York University, Ontario, Canada M3J 1P3 ${ }^{a}$ 
${ }^{1}$ also affiliated with University College London, United Kingdom

2 now at University of Salerno, Italy

3 also working at Max Planck Institute, Munich, Germany

${ }^{4}$ now at Institute of Aviation, Warsaw, Poland

${ }^{5}$ supported by the research grant No. 1 P03B 04529 (2005-2008)

6 This work was supported in part by the Marie Curie Actions Transfer of Knowledge project COCOS (contract MTKD-CT-2004-517186)

${ }^{7}$ now at DESY group FEB, Hamburg, Germany

8 also at Moscow State University, Russia

${ }^{9}$ now at University of Liverpool, UK

10 on leave of absence at CERN, Geneva, Switzerland

11 now at CERN, Geneva, Switzerland

12 also at Institut of Theoretical and Experimental Physics, Moscow, Russia

13 also at INP, Cracow, Poland

14 also at FPACS, AGH-UST, Cracow, Poland

15 partially supported by Warsaw University, Poland

16 partly supported by Moscow State University, Russia

17 also affiliated with DESY, Germany

18 also at University of Tokyo, Japan

19 now at Kobe University, Japan

20 supported by DESY, Germany

21 partly supported by Russian Foundation for Basic Research grant No. 05-02-39028NSFC-a

22 STFC Advanced Fellow

23 nee Korcsak-Gorzo

${ }^{24}$ This material was based on work supported by the National Science Foundation, while working at the Foundation.

25 now at University of Kansas, Lawrence, USA

26 also at Max Planck Institute, Munich, Germany, Alexander von Humboldt Research Award

27 now at KEK, Tsukuba, Japan

28 now at Nagoya University, Japan

29 member of Department of Radiological Science, Tokyo Metropolitan University, Japan

30 now at SunMelx Co. Ltd., Tokyo, Japan

31 now at University of Bonn, Germany

32 also at Hamburg University, Inst. of Exp. Physics, Alexander von Humboldt Research Award and partially supported by DESY, Hamburg, Germany

33 also at Łódź University, Poland

${ }^{34}$ member of Łódź University, Poland

35 now at Lund University, Lund, Sweden

$\dagger$ deceased 
a supported by the Natural Sciences and Engineering Research Council of Canada (NSERC)

$b$ supported by the German Federal Ministry for Education and Research (BMBF), under contract Nos. 05 HZ6PDA, 05 HZ6GUA, 05 HZ6VFA and 05 HZ4KHA

c supported in part by the MINERVA Gesellschaft für Forschung GmbH, the Israel Science Foundation (grant No. 293/02-11.2) and the US-Israel Binational Science Foundation

$d \quad$ supported by the Israel Science Foundation

$e$ supported by the Italian National Institute for Nuclear Physics (INFN)

$f$ supported by the Japanese Ministry of Education, Culture, Sports, Science and Technology (MEXT) and its grants for Scientific Research

$g$ supported by the Korean Ministry of Education and Korea Science and Engineering Foundation

$h \quad$ supported by the Netherlands Foundation for Research on Matter (FOM)

$i$ supported by the Polish State Committee for Scientific Research, project No. DESY/256/2006 - 154/DES/2006/03

$j$ partially supported by the German Federal Ministry for Education and Research $(\mathrm{BMBF})$

$k$ supported by RF Presidential grant N 1456.2008.2 for the leading scientific schools and by the Russian Ministry of Education and Science through its grant for Scientific Research on High Energy Physics

$l$ supported by the Spanish Ministry of Education and Science through funds provided by CICYT

$m$ supported by the Science and Technology Facilities Council, UK

$n$ supported by the US Department of Energy

$o$ supported by the US National Science Foundation. Any opinion, findings and conclusions or recommendations expressed in this material are those of the authors and do not necessarily reflect the views of the National Science Foundation.

$p \quad$ supported by the Polish Ministry of Science and Higher Education as a scientific project (2006-2008)

$q$ supported by FNRS and its associated funds (IISN and FRIA) and by an Inter-University Attraction Poles Programme subsidised by the Belgian Federal Science Policy Office

$r$ supported by an FRGS grant from the Malaysian government 


\section{Introduction}

The inclusive $e^{ \pm} p$ deep inelastic scattering (DIS) cross section can, at low virtuality of the exchanged boson, $Q^{2}$, be expressed in terms of the two structure functions, $F_{2}$ and $F_{L}$, as

$$
\frac{d^{2} \sigma^{e^{ \pm} p}}{d x d Q^{2}}=\frac{2 \pi \alpha^{2} Y_{+}}{x Q^{4}}\left[F_{2}\left(x, Q^{2}\right)-\frac{y^{2}}{Y_{+}} F_{L}\left(x, Q^{2}\right)\right]=\frac{2 \pi \alpha^{2} Y_{+}}{x Q^{4}} \tilde{\sigma}\left(x, Q^{2}, y\right)
$$

where $\alpha$ is the fine structure constant, $x$ is the Bjorken scaling variable, $y$ is the inelasticity and $Y_{+}=1+(1-y)^{2}$. The quantity $\tilde{\sigma}$ is referred to as the reduced cross section. The magnitude of $F_{L}$ is proportional to the absorption cross section of longitudinally polarised virtual photons by protons, $F_{L} \propto \sigma_{L}$, while $F_{2}$ includes also the absorption cross section for transversely polarised virtual photons, $F_{2} \propto\left(\sigma_{T}+\sigma_{L}\right)$. At low values of $x$ and small $\sigma_{L}$, the ratio $R=F_{L} /\left(F_{2}-F_{L}\right) \approx \sigma_{L} / \sigma_{T}$ gives the relative strength of the two components.

HERA measurements of the reduced $e p$ DIS cross section and $F_{2}[1,2]$ provide the strongest constraints on the proton parton distribution functions (PDFs) at low $x$. Within the DGLAP formalism [3], $F_{2}$ at low $x$ is dominated by the $q \bar{q}$ sea distributions while the scaling violations of $F_{2}$ reflect the gluon distribution, $g\left(x, Q^{2}\right)$, via a convolution with the splitting function $P_{q g}(x), \partial F_{2} / \partial \ln Q^{2} \sim \alpha_{s}\left(Q^{2}\right) P_{q g}(x) \otimes x g\left(x, Q^{2}\right)$, where $\alpha_{s}$ is the strong coupling constant.

The published values of $F_{2}$ at low $x$ at HERA required assumptions to be made about $F_{L}$. Moreover, gluon distributions extracted from scaling violations are dependent on the formalism [4] and the order of perturbative expansion [5] used to calculate the splitting functions. Measurements of the reduced cross section at fixed $\left(x, Q^{2}\right)$ and different $y$ allow $F_{2}$ and $F_{L}$ to be extracted simultaneously, thereby eliminating the assumptions about $F_{L}$ when extracting $F_{2}$. Furthermore, a direct measurement of $F_{L}$, which is strongly correlated to the gluon density [6], provides an important consistency check of the formalism.

A model-independent determination of $F_{L}$ requires the reduced cross section to be measured at fixed values of $x$ and $Q^{2}$ for multiple centre-of-mass energies (varying $y$ values). This method has been previously used to extract $F_{L}$ in fixed-target experiments [7-10] and recently by the $\mathrm{H} 1$ collaboration [11]. The $\mathrm{H} 1$ collaboration has also applied extrapolation methods to determine $F_{L}[12]$.

In this paper, the first ZEUS measurement of $F_{L}$ is presented as well as the most precise ZEUS measurement of $F_{2}$ in the kinematic region studied. Comparisons of theoretical predictions with the data are also presented. 


\section{Experimental method}

The values of $F_{2}$ and $F_{L}$ were extracted at fixed $x$ and $Q^{2}$ by fitting a straight line to the values of $\tilde{\sigma}$ against $y^{2} / Y_{+}$in the so-called Rosenbluth plot [13]. The method is based on Eq. 1, which implies that $F_{2}\left(x, Q^{2}\right)=\tilde{\sigma}\left(x, Q^{2}, y=0\right)$ and $F_{L}\left(x, Q^{2}\right)=$ $-\partial \tilde{\sigma}\left(x, Q^{2}, y\right) / \partial\left(y^{2} / Y_{+}\right)$, which in turn implies the need for data at fixed $\left(x, Q^{2}\right)$ and different $y$. In ep collisions, this can be achieved by varying the centre-of-mass energy $\sqrt{s}=\sqrt{Q^{2} / x y}$.

The precision of this procedure depends on the range available in $y^{2} / Y_{+}$. This was maximised by collecting data at the nominal HERA energy, $\sqrt{s}=318 \mathrm{GeV}$, and at $\sqrt{s}=225 \mathrm{GeV}$, the lowest attainable energy with adequate instantaneous luminosity. An intermediate data set was collected at $\sqrt{s}=251 \mathrm{GeV}$.

The variation of $\sqrt{s}$ was achieved by varying the proton beam energy, $E_{p}$, while keeping the electron beam energy constant, $E_{e}=27.5 \mathrm{GeV}$. Data were collected in 2006 and 2007 with $E_{p}=920,575$ and $460 \mathrm{GeV}$, referred to respectively as the high- (HER), medium- (MER) and low-energy-running (LER) samples. The corresponding integrated luminosities of the HER, MER and LER samples are 44.5, 7.1 and $13.9 \mathrm{pb}^{-1}$, respectively.

\section{$3 \quad$ Experimental apparatus}

A detailed description of the ZEUS detector can be found elsewhere [14]. A brief outline of the components most relevant for this analysis is given below.

In the kinematic range of the analysis, charged particles were tracked in the central tracking detector (CTD) [15] and the microvertex detector (MVD) [16]. These components operated in a magnetic field of $1.43 \mathrm{~T}$ provided by a thin superconducting solenoid. The CTD drift chamber covered the polar-angle ${ }^{1}$ region $15^{\circ}<\theta<164^{\circ}$. The MVD silicon tracker consisted of a barrel (BMVD) and a forward (FMVD) section. The BMVD provided polar-angle coverage for tracks with three measurements from $30^{\circ}$ to $150^{\circ}$. The FMVD extended the polar-angle coverage in the forward region to $7^{\circ}$.

The high-resolution uranium-scintillator calorimeter (CAL) [17] consisted of three parts: the forward (FCAL), the barrel (BCAL) and the rear (RCAL) calorimeters. Each part was subdivided transversely into towers and longitudinally into one electromagnetic section (EMC) and either one (in RCAL) or two (in BCAL and FCAL) hadronic sections (HAC).

\footnotetext{
${ }^{1}$ The ZEUS coordinate system is a right-handed Cartesian system, with the $Z$ axis pointing in the proton beam direction, referred to as the "forward direction", and the $X$ axis pointing towards the centre of HERA. The coordinate origin is at the nominal interaction point.
} 
The smallest subdivision of the calorimeter was called a cell. The CAL energy resolutions, as measured under test-beam conditions, were $\sigma(E) / E=0.18 / \sqrt{E}$ for electrons and $\sigma(E) / E=0.35 / \sqrt{E}$ for hadrons, with $E$ in $\mathrm{GeV}$.

The rear hadron-electron separator (RHES) [18] consisted of a layer of approximately $10000\left(3 \times 3 \mathrm{~cm}^{2}\right)$ silicon-pad detectors inserted in the RCAL at a depth of approximately 3 radiation lengths. The small-angle rear tracking detector (SRTD) [19] was attached to the front face of the RCAL and consisted of two planes of scintillator strips.

The small tungsten-scintillator calorimeter located approximately $6 \mathrm{~m}$ from the interaction point in the rear direction was referred to as the "6m-tagger" [20]. For scattered electrons in the energy range from 4.1 to $7.4 \mathrm{GeV}$, the acceptance was very close to one with very high purity.

The luminosity was measured using the Bethe-Heitler reaction $e p \rightarrow e \gamma p$ by a luminosity detector which consisted of an independent lead-scintillator calorimeter [21] and a magnetic spectrometer [22] system. The fractional systematic uncertainty on the measured luminosity was $2.6 \%$.

\section{Event reconstruction and selection}

The kinematic region studied spanned $0.09<y<0.78$ and $20<Q^{2}<130 \mathrm{GeV}^{2}$, corresponding to $5 \times 10^{-4}<x<0.007$. The event kinematics were evaluated based on the reconstruction of the scattered electron [23] using

$$
\begin{gathered}
y_{e}=1-\frac{E_{e}^{\prime}}{2 E_{e}}\left(1-\cos \theta_{e}\right), \\
Q_{e}^{2}=2 E_{e}^{\prime} E_{e}\left(1+\cos \theta_{e}\right),
\end{gathered}
$$

where $\theta_{e}$ and $E_{e}^{\prime}$ are the polar angle and energy of the scattered electron, respectively.

Electrons were identified using a neural network based on the moments of the threedimensional shower profile of clusters found in the CAL [24]. The quantity $E_{e}^{\prime}$ was reconstructed using the CAL, and $\theta_{e}$ was determined using the reconstructed interaction vertex and scattered electron position in the SRTD or, if outside the SRTD acceptance, in the RHES. In less than $2 \%$ of events, $\theta_{e}$ could not be determined in this way and such events were rejected.

A three-level trigger system was used to select events online [14, 25-27]. A dedicated trigger was developed providing high efficiency for high-y events [28]. The quantity $\delta \equiv \sum_{i}\left(E-p_{Z}\right)_{i}$ was used both in the trigger and in the offline analysis. The sum runs over all CAL energy deposits. Conservation of energy, $E$, and longitudinal momentum, 
$p_{Z}$, implies that $\delta=2 E_{e}=55 \mathrm{GeV}$. Undetected particles that escape through the forward beam-pipe hole contribute negligibly to $\delta$. Undetected particles that escape through the rear beam-pipe hole, such as the final-state electron in a photoproduction event, cause a substantial reduction in $\delta$. Events not originating from ep collisions often exhibit a very large $\delta$.

Events were selected offline if:

- $42<\delta<65 \mathrm{GeV}$;

- the reconstructed interaction vertex fulfilled $\left|Z_{\mathrm{vtx}}\right|<30 \mathrm{~cm}$;

- the energy of the most probable electron candidate satisfied $E_{e}^{\prime}>6 \mathrm{GeV}$;

- the event topology was not compatible with an elastic QED Compton (QEDC) event;

- the event timing was consistent with the HERA bunch structure;

- $y_{e}<0.95$ and $y_{\mathrm{JB}}>0.05$, where $y_{\mathrm{JB}}$ is the Jacquet-Blondel estimator [29] of $y$;

- $p_{T, h} / p_{T, e}>0.3$, where $p_{T, h}$ and $p_{T, e}$ refer to the transverse momentum of the hadronic system and electron candidate, respectively.

The projected path of the electron candidate was required to:

- exit the CTD at a radius $>20 \mathrm{~cm}$ and hence traverse the MVD fiducial volume and at least four CTD sense-wire layers, ensuring the possibility of identifying the track;

- enter the RCAL at a radius $<135 \mathrm{~cm}$, missing the inactive region between the RCAL and BCAL sections.

Hit information from the MVD and CTD was used to identify the tracks of the electron candidates. The procedure was based on the ratios of the number of observed to the maximum number of possible hits in the MVD and CTD, denoted $f_{\text {hit }}^{\mathrm{MVD}}$ and $f_{\text {hit }}^{\mathrm{CTD}}$, respectively:

- $f_{\text {hit }}^{\mathrm{MVD}}>0.45$;

- $f_{\text {hit }}^{\mathrm{CTD}}>0.6$.

This method was used because part of the kinematic region is outside the regular tracking capability of the MVD-CTD tracking system. After all cuts the HER, MER and LER samples contained 819168, 115719 and 205967 events, respectively.

\section{Cross section determination}

The reduced cross sections in a given $\left(x, Q^{2}\right)$ bin were calculated according to

$$
\tilde{\sigma}\left(x, Q^{2}\right)=\frac{N_{\mathrm{data}}-N_{\mathrm{MC}}^{\mathrm{bg}}}{N_{\mathrm{MC}}^{\mathrm{DIS}}} \tilde{\sigma}_{\mathrm{SM}}\left(x, Q^{2}\right),
$$


where $\tilde{\sigma}_{\mathrm{SM}}\left(x, Q^{2}\right)$ is the Standard Model electroweak Born-level reduced cross section and $N_{\text {data }}, N_{\mathrm{MC}}^{\mathrm{bg}}$ and $N_{\mathrm{MC}}^{\mathrm{DIS}}$ denote, respectively, the number of observed events in the data and the expected number of background and DIS events from the Monte Carlo (MC). The CTEQ5D [30] parameterisation of the proton PDF was used when calculating $\tilde{\sigma}_{\mathrm{SM}}\left(x, Q^{2}\right)$ as well as in the MC models when evaluating $N_{\mathrm{MC}}^{\mathrm{DIS}}$ and $N_{\mathrm{MC}}^{\mathrm{bg}}$. Specifically, the DIS signal processes were simulated using the DJANGOH 1.6 [31] MC model. After the full event selection, the background consisted almost entirely of photoproduction events. These were simulated using the PyтнiA 6.221 [32] MC model. The additional background components that were considered were elastic QEDC and mis-reconstructed low- $Q^{2}$ DIS, simulated using the Grape-Compton [33] and DJAngoh 1.6 MC models, respectively. The MC events were processed through a full simulation of the ZEUS detector and trigger system based on GEANT 3.21 [34].

The DJAngOH and Pythia samples included a diffractive component and first-order electroweak corrections. The diffractive and non-diffractive components of the DJANGOH sample were scaled to improve the description of the $\eta_{\max }$ distribution, where $\eta_{\max }$ is equal to the pseudorapidity of the most forward CAL energy deposit. The electroweak corrections were simulated using the Heracles 4.6 [31,35] MC model. Their uncertainty was evaluated by comparing the predictions from HERACLES to the higher-order predictions from Hector 1.0 [36]. The predictions were found to agree to within 0.5\%. The hadronic final state of the DJANGOH MC was simulated using the colour-dipole model of ARIADNE 4.12 [37] which uses the Lund string model of JETSET 7.4 [38] for the hadronisation.

In order to reduce the model dependence of the Monte Carlo prediction of the photoproduction component, the contribution from the direct subprocesses was enlarged to $9 \%$ in the inclusive Pythia sample while contributions by diffractive subprocesses were reduced accordingly. This procedure ensured that previous ZEUS results were reproduced $[39,40]$ and the predicted inclusive Pythia cross section remained unchanged. The predicted photoproduction cross section was then validated against a photoproduction data sample selected using the $6 \mathrm{~m}$-tagger. The predicted cross section was consistent with the data within the $\pm 10 \%$ total uncertainty on the data.

Figure 1 shows the distributions of the variables $E_{e}^{\prime}$ and $\theta_{e}$ within the HER, MER and LER data sets compared to the combined detector-level predictions from the MC models. The agreement is good in all cases. According to the MC models, the final data sample contained $97 \%$ DIS signal and $3 \%$ background events. The vast majority of the background events were found at low $Q^{2}$ and high $y$; in the most affected kinematic bin, the background fraction was $16 \%$.

The reduced cross sections, $\tilde{\sigma}$, were measured from the HER, MER and LER samples in the kinematic region $0.09<y<0.78$ and $20<Q^{2}<130 \mathrm{GeV}^{2}$. The $\tilde{\sigma}$ are given double differentially in $x$ and $Q^{2}$ in Tables $1-3$. The $\tilde{\sigma}$ are also shown at the 6 selected $Q^{2}$ values 
as functions of $x$ in Fig. 2. The cross sections have been compared to DGLAP-predictions based on the ZEUS-JETS PDF set [41], as well as the prediction for $F_{L} \equiv 0$. The QCD prediction with a non-zero $F_{L}$, describes the data well and is favoured over $F_{L} \equiv 0$.

\section{$6 \quad$ Systematic uncertainties}

The systematic uncertainty on the reduced cross sections due to the following sources were evaluated [28] (the numbers in the parentheses are the maximum uncertainty observed in any one of the reduced cross section bins):

- $\left\{\delta_{\gamma p}\right\}$, the $\pm 10 \%$ uncertainty on the level of photoproduction background $( \pm 1.5 \%)$;

- $\left\{\delta_{E_{e}}\right\}$, the electron energy-scale uncertainty of $\pm 0.5 \%$ for $E_{e}^{\prime}>20 \mathrm{GeV}$, increasing to $\pm 1.9 \%$ at $E_{e}^{\prime}=6 \mathrm{GeV}( \pm 2 \%)$;

- $\left\{\delta_{E_{h}}\right\}$, the $\pm 2 \%$ hadronic energy-scale uncertainty $( \pm 2.5 \%)$;

- $\left\{\delta_{e I D}\right\}$, the uncertainty on the electron-finding efficiency, evaluated by loosening (tightening) the criterion applied to the output of the neural network used to select electron candidates, both for data and $\mathrm{MC}( \pm 1 \%)$;

- $\left\{\delta_{d x}, \delta_{d y}\right\}$, the SRTD and HES position uncertainty of $\pm 2 \mathrm{~mm}$ in both the horizontal and vertical directions $( \pm 0.75 \%)$;

- $\left\{\delta_{\mathrm{MVD}}, \delta_{\mathrm{CTD}}\right\}$, the uncertainty on the hit-finding efficiency, evaluated by loosening (tightening) the hit fraction criteria, both for data and $\mathrm{MC}( \pm 3 \%)$;

- $\left\{\delta_{\text {diff }}\right\}$, the $\pm 10 \%$ uncertainty on the scale factors applied to the diffractive DJANGOH component $( \pm 0.5 \%)$.

The one-standard-deviation systematic uncertainties due to each source are listed in Tables 1-3 for the reduced cross sections at the three different centre-of-mass energies. All of the uncertainties are symmetric. They are quoted with a sign indicating how the reduced cross sections would vary given an upwards variation in the electron or hadronic energy scales, the SRTD and HES positions, the photoproduction cross section or the diffractive scale factors, or looser selection criteria on the neural network output or MVD or CTD hit fractions.

The total uncertainty on the normalisation included

- the luminosity uncertainty, which was $\pm 2.6 \%$ for all three data sets, of which $\pm 1 \%$ was uncorrelated between the data sets;

- the uncertainty on simulating the interaction-vertex distribution, evaluated by comparing the ratio of the number of events with $\left|Z_{\mathrm{vtx}}\right| \leq 30 \mathrm{~cm}$ and $\left|Z_{\mathrm{vtx}}\right|>30 \mathrm{~cm}$ in data and $\mathrm{MC}( \pm 0.3 \%)$; 
- the trigger-efficiency uncertainty $( \pm 0.5 \%)$.

The luminosity, vertex-distribution and trigger-efficiency uncertainties are perfectly correlated between bins and hence, when added in quadrature, constitute a total normalisation uncertainty of $\pm 2.7 \%$, of which $\pm 2.5 \%$ was correlated between the running periods and $\pm 1.1 \%$ uncorrelated. The uncertainty due the electroweak corrections was found to be negligible.

The total systematic uncertainty in each bin, formed by adding the individual uncertainties in quadrature, is also given in Tables 1-3. This sum also includes the statistical uncertainty due to the combined MC sample $\left\{\delta_{u n c}\right\}$ and is the only systematic source that is considered to be uncorrelated between bins. This total systematic uncertainty does not include the total normalisation uncertainty. Propagation of the systematic uncertainties to $F_{L}, F_{2}$ and $R$ is described in the next section.

\section{$7 \quad$ Extraction of $F_{L}, F_{2}$ and $R$}

In order to extract $F_{L}, F_{2}$ and $R$ a different binning scheme than that given in Tables 1-3 was applied to the reduced cross sections. Bins in $y$ were chosen such that, for each of the $6 Q^{2}$ bins, there were 3 values of $x$ at which the reduced cross sections were measured from all three data sets. This removed the need to interpolate the data between different points in the $\left(x, Q^{2}\right)$ plane. The structure functions were extracted by performing a simultaneous fit to these 54 measured cross section values using Eq. 1. Prior to fitting, the three data sets were normalised to their luminosity-weighted average in the restricted kinematic region, $y<0.3$, where the contribution to the reduced cross sections from $F_{L}$ is small. This procedure resulted in scaling the data by factors of $1.0027 \pm 0.0027,0.9869 \pm 0.0051$ and $0.9997 \pm 0.0039$, for the HER, MER and LER data sets, respectively. The spread of these factors is consistent with the uncorrelated part of the total normalisation uncertainty of $1.1 \%$.

To extract $F_{L}$ and $F_{2}, 48$ parameters were fit simultaneously: $18 F_{2}$ and $18 F_{L}$ values for the $18\left(x, Q^{2}\right)$ points; 3 relative normalisation factors for the HER, MER and LER data sets and 9 global shifts of systematic uncertainties $\left(\delta_{\gamma p}, \delta_{E_{e}}, \delta_{E_{h}}, \delta_{e I D}, \delta_{d x}, \delta_{d y}, \delta_{\mathrm{MVD}}, \delta_{\mathrm{CTD}}\right.$, $\left.\delta_{\text {diff }}\right)$. The three normalisation factors allowed for variations of the relative normalisation factors within their remaining uncertainties (see above). The nine global shifts allowed for changes in the central values of $\tilde{\sigma}$ in a correlated manner across the $\left(x, Q^{2}\right)$ plane according to the uncertainties listed. The probability distributions for the shifts of the systematic sources and the relative normalisations were taken to be Gaussian, with standard deviations equal to the corresponding systematic uncertainty. The probability distributions for the cross sections at each $\left(x, Q^{2}\right)$ point were also taken to be Gaussian with standard 
deviations given by $\delta_{\text {stat }}$ and $\delta_{\text {unc }}$ added in quadrature. The fit was performed within the BAT (Bayesian Analysis Toolkit) package [42] which, using a Markov chain MC, scans the full posterior probability density function in the 48-dimensional parameter space.

Initially, the $F_{L}$ and $F_{2}$ parameters were left unconstrained and flat prior probabilities were assumed. The results are given in Table 4, and are labelled with the superscript (1). The values quoted in the table were evaluated at the point where the probability density function attains its global maximum. The uncertainty ranges correspond to minimal $68 \%$ probability intervals. These ranges represent the full experimental uncertainty, which comprises statistical as well as systematic uncertainties. The fitted shifts, representing the correlated variation of the data points according to relative normalisation and correlated systematic uncertainties, are typically within 0.1 and at most 0.5 standard deviations of the normalisation or systematic uncertainties. The $F_{2}$ values typically have uncertainties of 0.03 , while the $F_{L}$ values have uncertainties ranging from 0.1 to 0.2 . These $F_{2}$ measurements are the most precise available from the ZEUS collaboration in the kinematic region studied here. The results are shown in Fig. 3 together with predictions from the ZEUS-JETS PDF fit. Good agreement is observed.

Applying constrained priors $F_{2} \geq 0$ and $0 \leq F_{L} \leq F_{2}$ in the fitting gave marginally different results as seen in Table 4 (results are denoted with the superscript (2)). For example, the most probable value for $F_{L}$ at $Q^{2}=45 \mathrm{GeV}^{2}$ and $x=0.00153$ is now 0 , in which case, a $68 \%$ probability upper limit is given.

Further fits to the data were performed to extract $F_{L}\left(Q^{2}\right), R\left(Q^{2}\right)$, and a single overall value of $R$ for the full data set. In each case, the same fitting procedure as described above was used, but with a reduced number of parameters. To extract $F_{L}\left(Q^{2}\right)$, first $r\left(Q^{2}\right)$ was fitted, where $r=F_{L} / F_{2}$. In fitting $r\left(Q^{2}\right)$, a single value of $r$ was taken for all $x$ points in the same $Q^{2}$ bin. Only a weak dependence of $r$ on $x$ in a restricted $x$ range is expected in the NLO DGLAP formalism as well as in phenomenological models. The data supports this prediction. Flat prior distributions for $r\left(Q^{2}\right)$ were assumed and both unconstrained and constrained fits were made, with $r\left(Q^{2}\right) \geq 0$ enforced in the latter. The value of $F_{L}\left(Q^{2}\right)$ was then evaluated as $F_{L}\left(x_{i}, Q^{2}\right)=r\left(Q^{2}\right) F_{2}\left(x_{i}, Q^{2}\right)$, where for each $Q^{2}$ point, $x_{i}$ was chosen such that $Q^{2} / x_{i}$ was constant, which for $\sqrt{s}=225 \mathrm{GeV}$, corresponds to $y=0.71$. The results are given in Table 5 and the unconstrained values are shown in Fig. 4a. These data are in good agreement with the results obtained by the H1 collaboration [11].

Values of $R\left(Q^{2}\right)$ and an overall value of $R$ were extracted with flat prior distributions. Both unconstrained and constrained fits were made. In the latter, it was required that $R\left(Q^{2}\right) \geq 0$ and $R \geq 0$. The results from both fits are given in Table 5 and the unconstrained $R\left(Q^{2}\right)$ values are shown in Fig. 4b. The uncertainty in the overall $R$ is not reduced as much as might be expected compared to the uncertainties on $R\left(Q^{2}\right)$ due to the 
correlation between the values at different $Q^{2}$. The value of $R$ from both the unconstrained and constrained fits was $R=0.18_{-0.05}^{+0.07}$.

Figures $4 \mathrm{a}$ and $4 \mathrm{~b}$ also show a comparison of the data with predictions based on the ZEUS-JETS and CTEQ6.6 [43] NLO and MSTW08 [44] NLO and NNLO² fits. All these predictions are based on the DGLAP formalism. Also shown are predictions from the NLL BFKL resummation fit from Thorne and White (TW) [47], and the prediction from the impact-parameter-dependent dipole saturation model (b-Sat) of Kowalski and Watt based on DGLAP evolution of the gluon density [48]. All of the models are consistent with the data.

\section{Summary}

The first measurement of $F_{L}\left(x, Q^{2}\right)$ by the ZEUS collaboration is presented, as is the first measurement of $F_{2}\left(x, Q^{2}\right)$ at low $x$ that does not include assumptions about $F_{L}$. The $F_{2}$ values are the most precise available from the ZEUS collaboration in the kinematic region studied. The extraction of $F_{L}$ and $F_{2}$ was based on the reduced double-differential cross sections, $\tilde{\sigma}\left(x, Q^{2}\right)$, which were measured for $0.09<y<0.78$ and $20<Q^{2}<130 \mathrm{GeV}^{2}$ using data collected at $\sqrt{s}=318,251$ and $225 \mathrm{GeV}$. In addition, $F_{L}$ and the ratio, $R$, have been extracted as a function of $Q^{2}$. An overall value of $R=0.18_{-0.05}^{+0.07}$ was extracted for the entire kinematic region studied. A wide range of theoretical predictions agree with the measured $F_{L}$. The measurements provide strong evidence of a non-zero $F_{L}$.

\section{Acknowledgements}

We appreciate the contributions to the construction and maintenance of the ZEUS detector of many people who are not listed as authors. The HERA machine group and the DESY computing staff are especially acknowledged for their success in providing excellent operation of the collider and the data-analysis environment. We thank the DESY directorate for their strong support and encouragement.

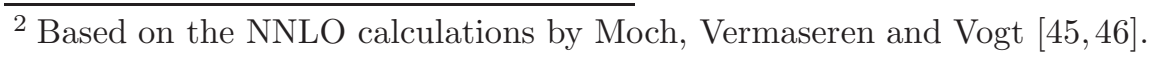




\section{References}

[1] ZEUS Coll., S. Chekanov et al., Eur. Phys. J. C 21, 443 (2001).

[2] H1 Coll., C. Adloff et al., Nucl. Phys. B 497, 3 (1997).

[3] Yu.L. Dokshitzer, Sov. Phys. JETP 46, 641 (1977);

V.N. Gribov and L.N. Lipatov, Sov. J. Nucl. Phys. 15, 438 (1972);

V.N. Gribov and L.N. Lipatov, Sov. J. Nucl. Phys. 15, 675 (1972);

G. Altarelli and G. Parisi, Nucl. Phys. B 126, 298 (1977).

[4] S. Catani, Z. Phys. C 75, 665 (1997).

[5] A.D. Martin, W.J. Stirling and R.S. Thorne, Phys. Lett. B 635, 305 (2006).

[6] G. Altarelli and G. Martinelli, Phys. Lett. B 76, 89 (1978).

[7] J.J. Aubert et al., Phys. Lett. B 121, 87 (1983).

[8] A.C. Benvenuti et al., Phys. Lett. B 223, 485 (1989).

[9] L.W. Whitlow et al., Phys. Lett. B 250, 193 (1990).

[10] M. Arneodo et al., Nucl. Phys. B 483, 3 (1997).

[11] H1 Coll., F.D. Aaron et al., Phys. Lett. B 665, 139 (2008).

[12] H1 Coll., C. Adloff et al., Phys. Lett. B 393, 452 (1997);

H1 Coll., C. Adloff et al., Eur. Phys. J. C 21, 33 (2001);

H1 Coll., C. Adloff et al., Eur. Phys. J. C 30, 1 (2003).

[13] M.N. Rosenbluth, Phys. Rev. 79, 615 (1950).

[14] ZEUS Coll., U. Holm (ed.), The ZEUS Detector. Status Report (unpublished), DESY (1993), available on http://www-zeus.desy.de/bluebook/bluebook.html.

[15] N. Harnew et al., Nucl. Inst. Meth. A 279, 290 (1989);

B. Foster et al., Nucl. Phys. Proc. Suppl. B 32, 181 (1993);

B. Foster et al., Nucl. Inst. Meth. A 338, 254 (1994).

[16] A. Polini et al., Nucl. Inst. Meth. A 581, 656 (2007).

[17] M. Derrick et al., Nucl. Inst. Meth. A 309, 77 (1991);

A. Andresen et al., Nucl. Inst. Meth. A 309, 101 (1991);

A. Caldwell et al., Nucl. Inst. Meth. A 321, 356 (1992);

A. Bernstein et al., Nucl. Inst. Meth. A 336, 23 (1993).

[18] A. Dwurazny et al., Nucl. Inst. Meth. A 277, 176 (1989).

[19] ZEUS Coll., A. Bamberger et al., Nucl. Inst. Meth. A 401, 63 (1997). 
[20] T. Gosau, Ph.D. Thesis, FB Physik, Univ. Hamburg, Germany, Report DESY-THESIS-2007-028, 2007;

M. Schröder, Diploma Thesis, FB Physik, Univ. Hamburg, Germany, Report DESY-THESIS-2008-039, 2008.

[21] J. Andruszków et al., Preprint DESY-92-066, DESY, 1992;

ZEUS Coll., M. Derrick et al., Z. Phys. C 63, 391 (1994);

J. Andruszków et al., Acta Phys. Pol. B 32, 2025 (2001).

[22] M. Helbich et al., Nucl. Inst. Meth. A 565, 572 (2006).

[23] K.C. Höger, Proc. Workshop on Physics at HERA, W. Buchmüller and G. Ingelman (eds.), Vol. 1, p. 43. Hamburg, Germany, DESY (1992).

[24] R. Sinkus and T. Voss, Nucl. Inst. Meth. A 391, 360 (1997);

H. Abramowicz, A. Caldwell and R. Sinkus, Nucl. Inst. Meth. A 365, 508 (1995).

[25] ZEUS Coll., J. Breitweg et al., Eur. Phys. J. C 1, 109 (1998).

[26] W.H. Smith et al., Nucl. Inst. Meth. A 355, 278 (1995).

[27] W.H. Smith, K. Tokushuku and L.W. Wiggers, Proc. Computing in High-Energy Physics (CHEP), Annecy, France, C. Verkerk and W. Wojcik (eds.), p. 222. CERN, Geneva, Switzerland (1992). Also in preprint DESY 92-150B.

[28] S. Shimizu, Ph.D. Thesis, University of Tokyo, Japan, 2009, KEK-report 2009-1.

[29] F. Jacquet and A. Blondel, Proceedings of the Study for an ep Facility for Europe, U. Amaldi (ed.), p. 391. Hamburg, Germany (1979). Also in preprint DESY 79/48.

[30] CTEQ Coll., H.L. Lai et al., Eur. Phys. J. C 12, 375 (2000).

[31] H. Spiesberger, HeRAClES and DJANGOH: event generation for ep interactions at HERA including radiative processes, DESY, Hamburg, Germany, 1998, available on http://www.desy.de/ ^hspiesb/djangoh.html.

[32] T. Sjöstrand et al., PYTHIA 6.2: Physics and manual, 2001, available on

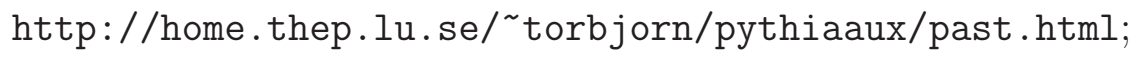

T. Sjöstrand et al., Comput. Phys. Commun. 135, 238 (2001).

[33] T. Abe, Comput. Phys. Commun. 136, 126 (2001).

[34] R. Brun et al., Geant3, CERN-DD/EE/84-1, 1987 (unpublished).

[35] H. Spiesberger, An event generator for ep interactions at HERA including radiative processes, Version 4.6, DESY, Hamburg, Germany, 1996, available on

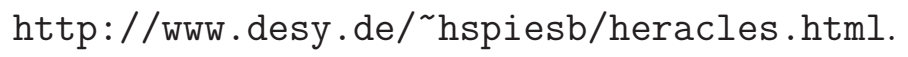

[36] A. Arbuzov et al., Comput. Phys. Commun. 94, 128 (1996).

[37] T. Sjöstrand, Comput. Phys. Commun. 39, 347 (1986). 
[38] T. Sjöstrand, Comput. Phys. Commun. 82, 74 (1994).

[39] ZEUS Coll. M. Derrick et al., Phys. Lett. B 293, 465 (1992).

[40] ZEUS Coll., S. Chekanov et al., Nucl. Phys. B 627, 3 (2002).

[41] ZEUS Coll. S. Chekanov et al., Eur. Phys. J. C 42, 1 (2005).

[42] A. Caldwell, D. Kollár and K. Kröninger, arXiv:0808.2552 [physics.data-an].

[43] P.M. Nadolsky et al., Phys. Rev. D 78, 013004 (2008).

[44] A.D. Martin et al., hep-ph/09010002.

[45] S. Moch, J.A.M. Vermaseren and A. Vogt, Phys. Lett. B 606, 123 (2005).

[46] J.A.M. Vermaseren, A. Vogt and S. Moch, Nucl. Phys. B 724, 3 (2005).

[47] C.D. White and R.S. Thorne, Phys. Rev. D 75, 034005 (2007).

[48] G. Watt and H. Kowalski, Phys. Rev. D 78, 014016 (2008);

H. Kowalski, L. Motyka and G. Watt, Phys. Rev. D 74, 074016 (2006);

H. Kowalski and D. Teaney, Phys. Rev. D 68, 114005 (2003). 


\begin{tabular}{|c|c|c|c|c|c|c|c|c|c|c|c|c|c|c|c|}
\hline $\begin{array}{r}Q^{2} \\
\left(\mathrm{GeV}^{2}\right)\end{array}$ & $x$ & $y$ & $\begin{array}{l}\tilde{\sigma}^{e^{+} p} \\
\text { HER }\end{array}$ & $\begin{array}{r}\delta_{\text {stat }} \\
(\%)\end{array}$ & $\begin{array}{r}\delta_{\text {sys }} \\
(\%)\end{array}$ & $\begin{array}{r}\delta_{\text {unc }} \\
(\%)\end{array}$ & $\begin{array}{l}\delta_{\gamma p} \\
(\%)\end{array}$ & $\begin{array}{l}\delta_{E_{e}} \\
(\%)\end{array}$ & $\begin{array}{l}\delta_{E_{h}} \\
(\%)\end{array}$ & $\begin{array}{r}\delta_{e I D} \\
(\%)\end{array}$ & $\begin{array}{l}\delta_{d x} \\
(\%)\end{array}$ & $\begin{array}{l}\delta_{d y} \\
(\%)\end{array}$ & $\begin{array}{r}\delta_{\mathrm{MVD}} \\
(\%)\end{array}$ & $\begin{array}{r}\delta_{\mathrm{CTD}} \\
(\%)\end{array}$ & $\begin{array}{r}\delta_{\text {diff }} \\
(\%)\end{array}$ \\
\hline 24 & $1.82 \cdot 10^{-3}$ & 0.13 & 1.057 & 1.0 & 2.5 & 0.9 & 0.0 & 1.0 & -0.8 & 0.0 & 0.8 & 0.3 & -0.4 & 1.7 & -0.3 \\
\hline 24 & $1.08 \cdot 10^{-3}$ & 0.22 & 1.234 & 0.8 & 2.4 & 0.8 & 0.0 & 1.6 & -0.2 & 0.0 & 0.2 & 0.3 & -0.6 & 1.4 & -0.3 \\
\hline 24 & $7.63 \cdot 10^{-4}$ & 0.31 & 1.321 & 0.8 & 2.2 & 0.7 & 0.0 & -0.2 & -0.2 & 0.0 & -0.3 & 0.1 & -1.8 & 1.0 & -0.4 \\
\hline 24 & $5.92 \cdot 10^{-4}$ & 0.40 & 1.410 & 0.8 & 1.6 & 0.7 & -0.1 & -0.4 & -0.3 & 0.3 & -0.7 & 0.1 & -0.3 & 1.0 & -0.4 \\
\hline 24 & $4.93 \cdot 10^{-4}$ & 0.48 & 1.453 & 0.8 & 1.5 & 0.8 & -0.3 & 0.3 & -0.5 & -0.1 & -0.2 & 0.1 & 0.3 & 0.9 & -0.4 \\
\hline 24 & $4.23 \cdot 10^{-4}$ & 0.56 & 1.448 & 0.9 & 2.7 & 1.0 & -0.6 & 0.4 & -0.8 & 0.6 & -0.4 & 0.2 & 1.0 & 1.9 & -0.5 \\
\hline 24 & $3.76 \cdot 10^{-4}$ & 0.63 & 1.452 & 1.1 & 2.7 & 1.2 & -0.8 & 0.1 & -1.4 & -0.6 & 0.3 & -0.1 & 0.7 & 1.4 & -0.5 \\
\hline 24 & $3.43 \cdot 10^{-4}$ & 0.69 & 1.489 & 1.3 & 3.6 & 1.5 & -1.2 & 0.5 & -2.4 & -0.6 & -0.3 & -0.1 & 0.7 & 1.2 & -0.6 \\
\hline 24 & $3.16 \cdot 10^{-4}$ & 0.75 & 1.521 & 1.5 & 5.1 & 2.0 & -2.0 & 0.4 & -3.9 & -0.7 & 0.5 & -0.4 & 1.3 & -0.5 & -0.7 \\
\hline 32 & $2.43 \cdot 10^{-3}$ & 0.13 & 1.027 & 0.6 & 1.8 & 0.6 & 0.0 & 1.1 & -0.6 & 0.0 & -0.5 & 0.1 & -0.8 & 0.7 & -0.2 \\
\hline 32 & $1.43 \cdot 10^{-3}$ & 0.22 & 1.209 & 0.6 & 2.0 & 0.6 & 0.0 & 1.5 & -0.2 & 0.0 & -0.6 & 0.1 & -0.5 & 0.8 & -0.3 \\
\hline 32 & $1.02 \cdot 10^{-3}$ & 0.31 & 1.331 & 0.7 & 1.6 & 0.6 & 0.0 & -0.1 & -0.2 & 0.1 & -0.1 & -0.1 & 0.4 & 1.3 & -0.4 \\
\hline 32 & $7.89 \cdot 10^{-4}$ & 0.40 & 1.388 & 0.8 & 1.5 & 0.7 & -0.1 & -0.6 & -0.2 & 0.2 & 0.4 & -0.1 & 0.1 & 1.0 & -0.4 \\
\hline 32 & $6.57 \cdot 10^{-4}$ & 0.48 & 1.435 & 0.9 & 1.7 & 0.8 & -0.2 & 0.0 & -0.3 & -0.5 & -0.5 & -0.1 & -0.8 & 0.8 & -0.4 \\
\hline 32 & $5.63 \cdot 10^{-4}$ & 0.56 & 1.504 & 1.0 & 2.2 & 1.0 & -0.4 & -0.4 & -0.7 & 0.1 & -0.1 & -0.2 & -1.5 & 0.6 & -0.5 \\
\hline 32 & $5.01 \cdot 10^{-4}$ & 0.63 & 1.465 & 1.3 & 2.6 & 1.5 & -0.7 & 1.2 & -1.3 & 0.2 & -0.1 & 0.1 & -0.6 & -0.4 & -0.5 \\
\hline 32 & $4.57 \cdot 10^{-4}$ & 0.69 & 1.522 & 1.4 & 3.1 & 1.6 & -0.8 & -0.9 & -1.8 & 0.3 & -0.3 & -0.2 & -1.3 & -0.4 & -0.6 \\
\hline 32 & $4.21 \cdot 10^{-4}$ & 0.75 & 1.470 & 1.7 & 4.4 & 2.2 & -1.7 & 1.5 & -2.5 & -0.9 & -0.4 & 0.3 & -1.0 & -0.7 & -0.5 \\
\hline 45 & $3.41 \cdot 10^{-3}$ & 0.13 & 0.984 & 0.6 & 1.6 & 0.5 & 0.0 & 0.9 & -0.7 & 0.0 & -0.1 & 0.1 & 0.1 & 1.0 & -0.2 \\
\hline 45 & $2.02 \cdot 10^{-3}$ & 0.22 & 1.151 & 0.6 & 2.0 & 0.5 & 0.0 & 1.5 & -0.2 & 0.0 & -0.2 & -0.2 & -0.6 & 0.9 & -0.3 \\
\hline 45 & $1.43 \cdot 10^{-3}$ & 0.31 & 1.253 & 0.7 & 1.4 & 0.6 & 0.0 & -0.2 & -0.1 & 0.0 & -0.3 & 0.1 & -0.7 & 0.8 & -0.4 \\
\hline 45 & $1.11 \cdot 10^{-3}$ & 0.40 & 1.376 & 0.9 & 1.3 & 0.8 & -0.1 & -0.5 & -0.2 & -0.1 & -0.4 & 0.2 & 0.1 & 0.7 & -0.4 \\
\hline 45 & $9.24 \cdot 10^{-4}$ & 0.48 & 1.408 & 1.0 & 1.8 & 0.9 & -0.1 & -1.0 & -0.3 & -0.2 & 0.4 & 0.0 & 0.5 & 0.9 & -0.5 \\
\hline 45 & $7.92 \cdot 10^{-4}$ & 0.56 & 1.492 & 1.1 & 1.8 & 1.1 & -0.3 & -0.2 & -0.4 & -0.4 & 0.5 & 0.1 & 0.6 & 0.8 & -0.4 \\
\hline 45 & $7.04 \cdot 10^{-4}$ & 0.63 & 1.483 & 1.4 & 2.5 & 1.5 & -0.6 & 0.4 & -1.0 & -1.1 & 0.5 & -0.3 & 0.5 & 0.3 & -0.5 \\
\hline 45 & $6.43 \cdot 10^{-4}$ & 0.69 & 1.571 & 1.6 & 3.1 & 1.9 & -0.8 & -0.3 & -1.3 & 0.6 & 0.5 & 0.1 & 1.6 & -0.4 & -0.5 \\
\hline 45 & $5.92 \cdot 10^{-4}$ & 0.75 & 1.517 & 1.8 & 3.7 & 2.3 & -1.4 & 0.9 & -1.8 & 0.4 & -0.2 & 0.2 & -0.7 & -1.2 & -0.6 \\
\hline 60 & $4.55 \cdot 10^{-3}$ & 0.13 & 0.932 & 0.7 & 1.7 & 0.6 & 0.0 & 0.9 & -0.6 & 0.0 & -0.5 & -0.3 & -0.6 & 0.7 & -0.1 \\
\hline 60 & $2.69 \cdot 10^{-3}$ & 0.22 & 1.119 & 0.7 & 1.9 & 0.6 & 0.0 & 1.5 & -0.1 & 0.0 & -0.4 & 0.0 & -0.7 & 0.5 & -0.3 \\
\hline 60 & $1.91 \cdot 10^{-3}$ & 0.31 & 1.231 & 0.9 & 1.1 & 0.7 & 0.0 & 0.2 & -0.1 & 0.0 & -0.2 & 0.2 & 0.3 & 0.6 & -0.3 \\
\hline 60 & $1.48 \cdot 10^{-3}$ & 0.40 & 1.337 & 1.0 & 1.6 & 0.9 & 0.0 & -1.1 & -0.2 & 0.1 & -0.6 & -0.3 & 0.3 & 0.4 & -0.4 \\
\hline 60 & $1.23 \cdot 10^{-3}$ & 0.48 & 1.388 & 1.2 & 1.7 & 1.1 & -0.1 & -0.5 & -0.2 & 0.3 & 0.2 & 0.3 & -0.8 & -0.6 & -0.5 \\
\hline 60 & $1.06 \cdot 10^{-3}$ & 0.56 & 1.510 & 1.3 & 1.8 & 1.2 & -0.2 & -0.7 & -0.5 & 0.0 & 0.3 & 0.2 & -0.8 & 0.1 & -0.4 \\
\hline 60 & $9.39 \cdot 10^{-4}$ & 0.63 & 1.560 & 1.7 & 2.6 & 1.7 & -0.4 & 0.6 & -0.7 & 0.6 & -0.6 & 0.3 & -0.9 & -1.0 & -0.5 \\
\hline 60 & $8.57 \cdot 10^{-4}$ & 0.69 & 1.504 & 1.9 & 3.1 & 2.2 & -0.9 & 1.0 & -1.3 & -0.2 & 0.1 & 0.4 & -0.5 & -0.4 & -0.5 \\
\hline 60 & $7.89 \cdot 10^{-4}$ & 0.75 & 1.586 & 2.1 & 3.6 & 2.2 & -0.9 & -1.7 & -1.6 & -0.6 & -0.4 & -0.3 & -0.5 & -0.9 & -0.6 \\
\hline
\end{tabular}

Continued on Next Page. . 


\begin{tabular}{|c|c|c|c|c|c|c|c|c|c|c|c|c|c|c|c|}
\hline $\begin{array}{r}Q^{2} \\
\left(\mathrm{GeV}^{2}\right)\end{array}$ & $x$ & $y$ & $\begin{array}{l}\tilde{\sigma}^{e^{+} p} \\
\text { HER }\end{array}$ & $\begin{array}{r}\delta_{\text {stat }} \\
(\%)\end{array}$ & $\begin{array}{r}\delta_{\text {sys }} \\
(\%)\end{array}$ & $\begin{array}{r}\delta_{u n c} \\
(\%)\end{array}$ & $\begin{array}{l}\delta_{\gamma p} \\
(\%)\end{array}$ & $\begin{array}{l}\delta_{E_{e}} \\
(\%)\end{array}$ & $\begin{array}{l}\delta_{E_{h}} \\
(\%)\end{array}$ & $\begin{array}{r}\delta_{e I D} \\
(\%)\end{array}$ & $\begin{array}{l}\delta_{d x} \\
(\%)\end{array}$ & $\begin{array}{l}\delta_{d y} \\
(\%)\end{array}$ & $\begin{array}{r}\delta_{\mathrm{MVD}} \\
(\%)\end{array}$ & $\begin{array}{r}\delta_{\mathrm{CTD}} \\
(\%)\end{array}$ & $\begin{array}{r}\delta_{\text {diff }} \\
(\%)\end{array}$ \\
\hline 80 & $6.07 \cdot 10^{-3}$ & 0.13 & 0.884 & 0.8 & 1.6 & 0.6 & 0.0 & 0.9 & -0.7 & 0.0 & 0.1 & 0.3 & -0.7 & 0.5 & -0.1 \\
\hline 80 & $3.59 \cdot 10^{-3}$ & 0.22 & 1.071 & 0.8 & 1.7 & 0.7 & 0.0 & 1.5 & -0.2 & 0.0 & -0.3 & -0.1 & -0.1 & 0.1 & -0.2 \\
\hline 80 & $2.54 \cdot 10^{-3}$ & 0.31 & 1.204 & 1.0 & 1.2 & 0.8 & 0.0 & -0.2 & -0.1 & -0.3 & -0.1 & -0.1 & -0.6 & -0.2 & -0.3 \\
\hline 80 & $1.97 \cdot 10^{-3}$ & 0.40 & 1.273 & 1.2 & 1.2 & 1.0 & -0.1 & -0.4 & -0.1 & 0.0 & 0.0 & 0.1 & 0.2 & 0.1 & -0.4 \\
\hline 80 & $1.41 \cdot 10^{-3}$ & 0.56 & 1.463 & 1.5 & 1.9 & 1.4 & -0.2 & 0.5 & -0.3 & 0.2 & -0.6 & -0.2 & 0.3 & 0.5 & -0.5 \\
\hline 80 & $1.25 \cdot 10^{-3}$ & 0.63 & 1.517 & 1.9 & 2.1 & 1.8 & -0.1 & -0.6 & -0.5 & -0.3 & 0.4 & -0.1 & -0.3 & -0.2 & -0.5 \\
\hline 80 & $1.14 \cdot 10^{-3}$ & 0.69 & 1.419 & 2.2 & 3.2 & 2.5 & -0.8 & 0.1 & -0.8 & 0.3 & -0.1 & -0.5 & 0.4 & 1.3 & -0.4 \\
\hline 80 & $1.05 \cdot 10^{-3}$ & 0.75 & 1.436 & 2.5 & 4.6 & 3.2 & -1.2 & 1.9 & -1.9 & 0.6 & -0.4 & -0.2 & 0.9 & -0.9 & -0.5 \\
\hline 110 & $8.34 \cdot 10^{-3}$ & 0.13 & 0.837 & 0.9 & 1.3 & 0.8 & 0.0 & 0.7 & -0.7 & 0.0 & -0.2 & 0.2 & -0.1 & 0.2 & -0.1 \\
\hline 110 & $4.93 \cdot 10^{-3}$ & 0.22 & 1.005 & 1.0 & 1.7 & 0.9 & 0.0 & 1.4 & -0.2 & -0.1 & -0.2 & -0.2 & 0.1 & 0.1 & -0.2 \\
\hline 110 & $2.71 \cdot 10^{-3}$ & 0.40 & 1.240 & 1.4 & 1.5 & 1.2 & -0.1 & -0.7 & 0.1 & 0.0 & -0.3 & 0.1 & 0.2 & 0.2 & -0.4 \\
\hline 110 & $2.26 \cdot 10^{-3}$ & 0.48 & 1.300 & 1.6 & 1.9 & 1.5 & -0.2 & -0.4 & 0.0 & -0.1 & -0.4 & 0.3 & -0.8 & -0.3 & -0.4 \\
\hline 110 & $1.94 \cdot 10^{-3}$ & 0.56 & 1.420 & 1.8 & 2.0 & 1.7 & -0.2 & 0.6 & -0.2 & 0.1 & -0.7 & -0.2 & 0.0 & -0.5 & -0.4 \\
\hline 110 & $1.72 \cdot 10^{-3}$ & 0.63 & 1.409 & 2.3 & 3.2 & 2.4 & -0.4 & -1.6 & -0.3 & 0.5 & 0.5 & 0.1 & 0.3 & -0.7 & -0.4 \\
\hline 110 & $1.57 \cdot 10^{-3}$ & 0.69 & 1.584 & 2.6 & 3.5 & 2.8 & -0.7 & -0.8 & -0.6 & 0.7 & -0.2 & -0.3 & 0.4 & 1.4 & -0.4 \\
\hline 110 & $1.45 \cdot 10^{-3}$ & 0.75 & 1.717 & 4.1 & 5.5 & 4.6 & -0.9 & 1.5 & 0.9 & 1.2 & 0.8 & -0.1 & -0.4 & -1.7 & -0.4 \\
\hline
\end{tabular}

Table 1: The reduced cross section, $\tilde{\sigma}$, for the reaction $e^{+} p \rightarrow e^{+} X$ at $\sqrt{s}=318 \mathrm{GeV}$. The first three columns contain the bin centres in $Q^{2}, x$ and $y$, the next three contain the measured cross section, the statistical uncertainty and the total systematic uncertainty, respectively. The final ten columns list the uncorrelated, $\delta_{\text {unc }}$ and the bin-to-bin correlated uncertainties from each systematic source, $\delta_{\gamma p}, \delta_{E_{e}}, \delta_{E_{h}}, \delta_{e I D}$, $\delta_{d x}, \delta_{d y}, \delta_{\mathrm{MVD}}, \delta_{\mathrm{CTD}}, \delta_{\mathrm{diff}}$. For details, see Section 6. A further $\pm 2.7 \%$ systematic normalisation uncertainty is not included, of which $\pm 2.5 \%$ is correlated between the running periods and $\pm 1.1 \%$ is uncorrelated. 


\begin{tabular}{|c|c|c|c|c|c|c|c|c|c|c|c|c|c|c|c|}
\hline $\begin{array}{r}Q^{2} \\
\left(\mathrm{GeV}^{2}\right)\end{array}$ & $x$ & $y$ & $\begin{array}{l}\tilde{\sigma}^{e^{+} p} \\
\text { MER }\end{array}$ & $\begin{array}{r}\delta_{\text {stat }} \\
(\%)\end{array}$ & $\begin{array}{l}\delta_{\text {sys }} \\
(\%)\end{array}$ & $\begin{array}{r}\delta_{\text {unc }} \\
(\%)\end{array}$ & $\begin{array}{l}\delta_{\gamma p} \\
(\%)\end{array}$ & $\begin{array}{l}\delta_{E_{e}} \\
(\%)\end{array}$ & $\begin{array}{l}\delta_{E_{h}} \\
(\%)\end{array}$ & $\begin{array}{r}\delta_{e I D} \\
(\%)\end{array}$ & $\begin{array}{l}\delta_{d x} \\
(\%)\end{array}$ & $\begin{array}{l}\delta_{d y} \\
(\%)\end{array}$ & $\begin{array}{r}\delta_{\mathrm{MVD}} \\
(\%)\end{array}$ & $\begin{array}{r}\delta_{\mathrm{CTD}} \\
(\%)\end{array}$ & $\begin{array}{l}\delta_{\text {diff }} \\
(\%)\end{array}$ \\
\hline 24 & $2.91 \cdot 10^{-3}$ & 0.13 & 0.955 & 2.4 & 3.3 & 1.3 & 0.0 & 0.9 & -0.7 & 0.0 & 1.1 & 0.5 & -1.6 & 2.0 & -0.2 \\
\hline 24 & $1.72 \cdot 10^{-3}$ & 0.22 & 1.105 & 2.1 & 3.0 & 1.1 & 0.0 & 1.6 & -0.3 & 0.2 & 1.4 & -0.3 & -1.4 & 1.1 & -0.3 \\
\hline 24 & $1.22 \cdot 10^{-3}$ & 0.31 & 1.153 & 2.1 & 2.1 & 1.0 & 0.0 & -0.6 & -0.2 & 0.0 & -0.3 & -0.6 & -0.6 & 1.4 & -0.3 \\
\hline 24 & $9.47 \cdot 10^{-4}$ & 0.40 & 1.267 & 2.1 & 2.3 & 1.0 & -0.1 & 0.1 & -0.2 & 0.5 & 1.1 & 0.2 & 0.8 & 1.3 & -0.4 \\
\hline 24 & $7.89 \cdot 10^{-4}$ & 0.48 & 1.328 & 2.2 & 2.6 & 1.2 & -0.3 & -0.7 & -0.4 & -0.4 & -0.3 & -0.2 & 0.7 & 1.9 & -0.4 \\
\hline 24 & $6.76 \cdot 10^{-4}$ & 0.56 & 1.319 & 2.4 & 5.0 & 1.3 & -0.5 & 0.7 & -0.8 & 0.2 & -0.5 & 0.4 & 2.8 & 3.7 & -0.4 \\
\hline 24 & $6.01 \cdot 10^{-4}$ & 0.63 & 1.334 & 3.0 & 3.8 & 1.6 & -0.7 & -0.3 & -1.4 & -0.9 & 1.2 & 0.6 & 2.0 & 1.6 & -0.5 \\
\hline 24 & $5.49 \cdot 10^{-4}$ & 0.69 & 1.432 & 3.2 & 3.9 & 1.8 & -1.0 & -0.5 & -2.0 & -0.2 & -0.3 & 0.6 & 0.8 & 2.2 & -0.6 \\
\hline 24 & $5.05 \cdot 10^{-4}$ & 0.75 & 1.389 & 3.9 & 5.0 & 2.5 & -2.0 & -0.8 & -3.5 & 0.9 & 0.2 & 0.4 & -0.4 & 0.8 & -0.6 \\
\hline 32 & $3.88 \cdot 10^{-3}$ & 0.13 & 0.909 & 1.7 & 2.1 & 0.8 & 0.0 & 1.1 & -0.8 & 0.0 & -0.2 & -0.1 & -0.3 & 1.3 & -0.2 \\
\hline 32 & $2.29 \cdot 10^{-3}$ & 0.22 & 1.074 & 1.7 & 2.4 & 0.8 & 0.0 & 1.6 & -0.3 & 0.1 & -0.8 & 0.2 & -0.6 & 1.1 & -0.2 \\
\hline 32 & $1.63 \cdot 10^{-3}$ & 0.31 & 1.153 & 1.9 & 2.9 & 0.9 & -0.1 & -0.3 & -0.1 & 0.0 & 0.8 & 0.9 & 1.0 & 2.3 & -0.4 \\
\hline 32 & $1.26 \cdot 10^{-3}$ & 0.40 & 1.226 & 2.1 & 2.2 & 1.0 & -0.1 & -0.3 & -0.2 & 0.4 & 0.1 & 0.2 & 0.6 & 1.6 & -0.4 \\
\hline 32 & $1.05 \cdot 10^{-3}$ & 0.48 & 1.326 & 2.3 & 1.9 & 1.2 & -0.2 & -0.6 & -0.3 & -0.1 & 0.2 & 0.2 & -0.2 & 1.2 & -0.4 \\
\hline 32 & $9.01 \cdot 10^{-4}$ & 0.56 & 1.270 & 2.7 & 2.5 & 1.4 & -0.5 & 0.6 & -0.8 & -0.8 & -0.8 & -0.9 & -0.5 & 0.7 & -0.4 \\
\hline 32 & $8.01 \cdot 10^{-4}$ & 0.63 & 1.381 & 3.4 & 2.9 & 1.9 & -0.5 & 0.6 & -1.0 & 0.6 & -1.6 & -0.2 & 0.4 & 0.5 & -0.6 \\
\hline 32 & $7.32 \cdot 10^{-4}$ & 0.69 & 1.314 & 3.9 & 3.7 & 2.2 & -0.9 & 0.5 & -1.7 & 0.4 & 0.1 & -0.8 & -1.7 & 1.0 & -0.5 \\
\hline 32 & $6.73 \cdot 10^{-4}$ & 0.75 & 1.355 & 4.5 & 5.0 & 2.7 & -1.6 & 1.6 & -2.8 & -1.1 & 0.9 & -0.3 & -1.6 & 0.3 & -0.6 \\
\hline 45 & $5.46 \cdot 10^{-3}$ & 0.13 & 0.890 & 1.5 & 2.4 & 0.7 & 0.0 & 1.2 & -0.7 & 0.0 & -0.3 & 0.3 & 0.3 & 1.7 & -0.1 \\
\hline 45 & $3.23 \cdot 10^{-3}$ & 0.22 & 1.037 & 1.7 & 2.5 & 0.8 & 0.0 & 1.2 & -0.2 & 0.0 & -0.4 & 0.2 & 0.9 & 1.8 & -0.2 \\
\hline 45 & $2.29 \cdot 10^{-3}$ & 0.31 & 1.126 & 1.9 & 1.8 & 0.9 & 0.0 & 0.2 & -0.1 & -0.2 & -1.0 & -0.5 & -0.5 & 0.8 & -0.3 \\
\hline 45 & $1.77 \cdot 10^{-3}$ & 0.40 & 1.171 & 2.3 & 2.5 & 1.1 & -0.1 & -0.4 & 0.1 & 0.3 & -1.5 & -0.1 & 0.6 & 1.4 & -0.4 \\
\hline 45 & $1.48 \cdot 10^{-3}$ & 0.48 & 1.270 & 2.7 & 2.3 & 1.3 & -0.2 & -0.5 & -0.3 & 0.3 & 0.7 & 0.5 & 0.4 & 1.4 & -0.5 \\
\hline 45 & $1.27 \cdot 10^{-3}$ & 0.56 & 1.323 & 3.0 & 2.1 & 1.5 & -0.3 & -0.5 & -0.6 & 0.1 & 0.4 & -0.8 & -0.3 & 0.6 & -0.5 \\
\hline 45 & $1.13 \cdot 10^{-3}$ & 0.63 & 1.385 & 3.8 & 3.3 & 2.2 & -0.6 & -0.8 & -1.1 & 1.8 & 0.4 & 0.5 & 0.2 & 0.3 & -0.4 \\
\hline 45 & $1.03 \cdot 10^{-3}$ & 0.69 & 1.443 & 4.2 & 3.7 & 2.4 & -0.8 & -0.6 & -1.3 & 0.2 & 1.1 & 0.4 & 1.2 & 1.6 & -0.6 \\
\hline 45 & $9.47 \cdot 10^{-4}$ & 0.75 & 1.400 & 4.7 & 3.8 & 2.6 & -1.0 & 0.7 & -1.9 & -1.0 & 0.6 & 0.8 & 0.6 & 0.5 & -0.4 \\
\hline 60 & $7.28 \cdot 10^{-3}$ & 0.13 & 0.809 & 1.8 & 1.9 & 0.8 & 0.0 & 0.9 & -0.7 & 0.0 & -0.2 & -0.1 & 0.4 & 1.2 & -0.1 \\
\hline 60 & $4.30 \cdot 10^{-3}$ & 0.22 & 0.970 & 2.0 & 2.2 & 0.9 & 0.0 & 1.4 & -0.2 & 0.0 & -0.2 & 0.5 & 0.7 & 1.1 & -0.3 \\
\hline 60 & $3.05 \cdot 10^{-3}$ & 0.31 & 1.123 & 2.3 & 1.8 & 1.1 & 0.0 & -0.6 & -0.1 & 0.0 & -0.1 & -0.4 & 0.8 & 0.9 & -0.3 \\
\hline 60 & $2.37 \cdot 10^{-3}$ & 0.40 & 1.183 & 2.7 & 1.6 & 1.3 & 0.0 & -0.2 & -0.1 & 0.0 & 0.7 & -0.3 & -0.2 & 0.3 & -0.4 \\
\hline 60 & $1.97 \cdot 10^{-3}$ & 0.48 & 1.135 & 3.3 & 2.2 & 1.5 & -0.1 & 0.6 & -0.5 & 0.3 & 0.7 & -0.9 & -0.2 & -0.5 & -0.4 \\
\hline 60 & $1.69 \cdot 10^{-3}$ & 0.56 & 1.277 & 3.6 & 2.9 & 1.8 & -0.3 & -1.0 & -0.3 & -0.1 & 1.0 & 0.7 & 1.4 & 0.7 & -0.4 \\
\hline 60 & $1.50 \cdot 10^{-3}$ & 0.63 & 1.417 & 4.4 & 3.0 & 2.3 & -0.3 & 1.3 & -0.4 & 0.2 & 0.6 & 0.4 & 0.8 & -0.3 & -0.5 \\
\hline 60 & $1.37 \cdot 10^{-3}$ & 0.69 & 1.300 & 5.2 & 4.4 & 3.1 & -1.0 & 1.8 & -0.7 & 0.8 & 1.5 & -1.2 & 0.5 & -0.4 & -0.4 \\
\hline 60 & $1.26 \cdot 10^{-3}$ & 0.75 & 1.446 & 5.6 & 4.8 & 3.1 & -0.9 & -1.4 & -2.1 & -0.4 & -1.8 & -0.7 & 0.4 & -1.1 & -0.5 \\
\hline
\end{tabular}

Continued on Next Page... 


\begin{tabular}{|c|c|c|c|c|c|c|c|c|c|c|c|c|c|c|c|}
\hline $\begin{array}{r}Q^{2} \\
\left(\mathrm{GeV}^{2}\right)\end{array}$ & $x$ & $y$ & $\begin{array}{l}\tilde{\sigma}^{e^{+} p} \\
\text { MER }\end{array}$ & $\begin{array}{r}\delta_{\text {stat }} \\
(\%)\end{array}$ & $\begin{array}{r}\delta_{\text {sys }} \\
(\%)\end{array}$ & $\begin{array}{r}\delta_{\text {unc }} \\
(\%)\end{array}$ & $\begin{array}{l}\delta_{\gamma p} \\
(\%)\end{array}$ & $\begin{array}{l}\delta_{E_{e}} \\
(\%)\end{array}$ & $\begin{array}{l}\delta_{E_{h}} \\
(\%)\end{array}$ & $\begin{array}{r}\delta_{e I D} \\
(\%)\end{array}$ & $\begin{array}{l}\delta_{d x} \\
(\%)\end{array}$ & $\begin{array}{l}\delta_{d y} \\
(\%)\end{array}$ & $\begin{array}{r}\delta_{\mathrm{MVD}} \\
(\%)\end{array}$ & $\begin{array}{r}\delta_{\mathrm{CTD}} \\
(\%)\end{array}$ & $\begin{array}{r}\delta_{\text {diff }} \\
(\%)\end{array}$ \\
\hline 80 & $9.71 \cdot 10^{-3}$ & 0.13 & 0.751 & 2.1 & 1.7 & 1.0 & 0.0 & 0.8 & -0.7 & 0.0 & 0.7 & 0.2 & -0.3 & 0.6 & -0.1 \\
\hline 80 & $5.74 \cdot 10^{-3}$ & 0.22 & 0.947 & 2.3 & 1.9 & 1.1 & 0.0 & 1.3 & -0.1 & 0.1 & -0.7 & -0.2 & -0.5 & 0.3 & -0.2 \\
\hline 80 & $4.07 \cdot 10^{-3}$ & 0.31 & 1.067 & 2.7 & 1.4 & 1.3 & 0.0 & 0.4 & 0.0 & 0.0 & 0.4 & -0.1 & -0.1 & 0.3 & -0.3 \\
\hline 80 & $3.16 \cdot 10^{-3}$ & 0.40 & 1.106 & 3.2 & 2.2 & 1.5 & 0.0 & -1.4 & -0.1 & 0.3 & -0.3 & 0.5 & 0.1 & 0.0 & -0.3 \\
\hline 80 & $2.63 \cdot 10^{-3}$ & 0.48 & 1.170 & 3.7 & 2.5 & 1.8 & -0.2 & -0.7 & -0.3 & -0.5 & -1.0 & 0.3 & -0.6 & -0.8 & -0.4 \\
\hline 80 & $2.25 \cdot 10^{-3}$ & 0.56 & 1.217 & 4.2 & 3.4 & 2.2 & -0.4 & -0.7 & -0.6 & 0.7 & -1.1 & -0.4 & -1.4 & -1.3 & -0.3 \\
\hline 80 & $2.00 \cdot 10^{-3}$ & 0.63 & 1.246 & 5.5 & 3.9 & 2.9 & -0.5 & 0.7 & -1.3 & 0.6 & -0.2 & -0.4 & 1.3 & 1.2 & -0.4 \\
\hline 80 & $1.83 \cdot 10^{-3}$ & 0.69 & 1.274 & 6.1 & 4.5 & 3.7 & -1.0 & 0.3 & -0.4 & 0.8 & -1.2 & 0.7 & -0.5 & 1.5 & -0.5 \\
\hline 80 & $1.68 \cdot 10^{-3}$ & 0.75 & 1.461 & 6.3 & 5.3 & 3.6 & -0.8 & -2.4 & -1.8 & -1.2 & 0.4 & -0.1 & -0.6 & -1.6 & -0.6 \\
\hline 110 & $1.33 \cdot 10^{-2}$ & 0.13 & 0.730 & 2.4 & 2.1 & 1.1 & 0.0 & 0.8 & -0.6 & 0.1 & -0.7 & 0.2 & 1.0 & 0.7 & -0.1 \\
\hline 110 & $7.89 \cdot 10^{-3}$ & 0.22 & 0.835 & 2.8 & 2.3 & 1.3 & 0.0 & 1.4 & -0.2 & 0.1 & 1.1 & 0.6 & -0.2 & -0.4 & -0.1 \\
\hline 110 & $5.60 \cdot 10^{-3}$ & 0.31 & 0.971 & 3.2 & 1.7 & 1.5 & 0.0 & 0.4 & -0.1 & -0.1 & 0.2 & 0.4 & 0.1 & -0.3 & -0.2 \\
\hline 110 & $4.34 \cdot 10^{-3}$ & 0.40 & 1.078 & 3.8 & 2.5 & 1.8 & 0.0 & -0.6 & -0.1 & 0.6 & -0.6 & -0.7 & -0.5 & -1.1 & -0.3 \\
\hline 110 & $3.62 \cdot 10^{-3}$ & 0.48 & 1.116 & 4.4 & 3.5 & 2.3 & -0.2 & -2.3 & 0.0 & 0.0 & -0.3 & -0.3 & -1.1 & 0.8 & -0.4 \\
\hline 110 & $3.10 \cdot 10^{-3}$ & 0.56 & 1.192 & 5.0 & 4.5 & 2.7 & -0.5 & -1.0 & -0.4 & -1.5 & 1.3 & -0.4 & 0.6 & 2.7 & -0.5 \\
\hline 110 & $2.75 \cdot 10^{-3}$ & 0.63 & 1.127 & 6.6 & 4.0 & 3.4 & -0.4 & 1.0 & -0.5 & 0.4 & -0.9 & 0.7 & 1.2 & 0.3 & -0.5 \\
\hline 110 & $2.51 \cdot 10^{-3}$ & 0.69 & 1.174 & 7.7 & 6.4 & 4.1 & -0.7 & -2.7 & -1.1 & 0.4 & -0.6 & -0.4 & -2.4 & 3.0 & -0.2 \\
\hline 110 & $2.31 \cdot 10^{-3}$ & 0.75 & 1.354 & 10.9 & 9.1 & 6.2 & -1.0 & 4.4 & -1.6 & 0.4 & 3.3 & 0.3 & -1.6 & -2.7 & -0.6 \\
\hline
\end{tabular}

Table 2: The reduced cross section, $\tilde{\sigma}$, for the reaction $e^{+} p \rightarrow e^{+} X$ at $\sqrt{s}=251 \mathrm{GeV}$.

Further details as described in caption of Table1. 


\begin{tabular}{|c|c|c|c|c|c|c|c|c|c|c|c|c|c|c|c|}
\hline $\begin{array}{r}Q^{2} \\
\left(\mathrm{GeV}^{2}\right)\end{array}$ & $x$ & $y$ & $\begin{array}{l}\tilde{\sigma}^{e^{+} p} \\
\text { LER }\end{array}$ & $\begin{array}{r}\delta_{\text {stat }} \\
(\%)\end{array}$ & $\begin{array}{r}\delta_{\text {sys }} \\
(\%)\end{array}$ & $\begin{array}{r}\delta_{\text {unc }} \\
(\%)\end{array}$ & $\begin{array}{l}\delta_{\gamma p} \\
(\%)\end{array}$ & $\begin{array}{l}\delta_{E_{e}} \\
(\%)\end{array}$ & $\begin{array}{l}\delta_{E_{h}} \\
(\%)\end{array}$ & $\begin{array}{r}\delta_{e I D} \\
(\%)\end{array}$ & $\begin{array}{l}\delta_{d x} \\
(\%)\end{array}$ & $\begin{array}{l}\delta_{d y} \\
(\%)\end{array}$ & $\begin{array}{r}\delta_{\mathrm{MVD}} \\
(\%)\end{array}$ & $\begin{array}{r}\delta_{\mathrm{CTD}} \\
(\%)\end{array}$ & $\begin{array}{r}\delta_{\text {diff }} \\
(\%)\end{array}$ \\
\hline 24 & $3.64 \cdot 10^{-3}$ & 0.13 & 0.864 & 1.8 & 2.7 & 0.9 & 0.0 & 1.2 & -0.8 & 0.0 & 0.7 & 0.7 & -1.2 & 1.4 & -0.1 \\
\hline 24 & $2.15 \cdot 10^{-3}$ & 0.22 & 1.043 & 1.6 & 2.8 & 0.8 & 0.0 & 1.7 & -0.2 & 0.0 & -0.6 & 0.3 & -0.6 & 1.8 & -0.2 \\
\hline 24 & $1.53 \cdot 10^{-3}$ & 0.31 & 1.136 & 1.5 & 2.0 & 0.7 & 0.0 & -0.4 & -0.2 & 0.0 & -0.4 & -0.3 & -1.3 & 1.2 & -0.4 \\
\hline 24 & $1.18 \cdot 10^{-3}$ & 0.40 & 1.184 & 1.6 & 2.0 & 0.7 & -0.1 & -0.7 & -0.3 & 0.2 & -1.0 & 0.3 & -0.6 & 1.2 & -0.4 \\
\hline 24 & $9.86 \cdot 10^{-4}$ & 0.48 & 1.195 & 1.7 & 2.7 & 0.8 & -0.2 & -0.2 & -0.4 & 0.1 & -0.3 & -0.2 & 1.4 & 2.1 & -0.4 \\
\hline 24 & $8.45 \cdot 10^{-4}$ & 0.56 & 1.260 & 1.7 & 2.0 & 0.9 & -0.3 & 0.7 & -0.7 & 0.4 & -0.8 & -0.2 & -0.8 & 0.7 & -0.5 \\
\hline 24 & $7.51 \cdot 10^{-4}$ & 0.63 & 1.256 & 2.2 & 3.1 & 1.3 & -0.8 & 0.3 & -1.5 & -0.7 & -0.3 & 0.5 & -1.8 & -0.8 & -0.5 \\
\hline 24 & $6.86 \cdot 10^{-4}$ & 0.69 & 1.260 & 2.5 & 3.5 & 1.6 & -1.3 & 1.3 & -1.9 & 0.3 & -0.3 & 0.5 & -0.4 & 1.3 & -0.6 \\
\hline 24 & $6.31 \cdot 10^{-4}$ & 0.75 & 1.247 & 2.9 & 5.6 & 2.1 & -2.0 & 0.6 & -4.1 & 0.8 & -1.1 & -0.9 & 1.2 & 0.6 & -0.6 \\
\hline 32 & $4.85 \cdot 10^{-3}$ & 0.13 & 0.848 & 1.3 & 1.9 & 0.6 & 0.0 & 1.1 & -0.8 & 0.0 & -0.4 & -0.2 & -0.6 & 1.0 & -0.1 \\
\hline 32 & $2.87 \cdot 10^{-3}$ & 0.22 & 0.977 & 1.3 & 2.1 & 0.6 & 0.0 & 1.6 & -0.2 & 0.0 & 0.2 & 0.3 & -0.5 & 1.1 & -0.2 \\
\hline 32 & $2.04 \cdot 10^{-3}$ & 0.31 & 1.083 & 1.4 & 1.4 & 0.6 & 0.0 & -0.4 & -0.2 & 0.1 & -0.5 & 0.3 & -0.1 & 0.9 & -0.3 \\
\hline 32 & $1.58 \cdot 10^{-3}$ & 0.40 & 1.153 & 1.6 & 1.2 & 0.7 & -0.1 & -0.5 & -0.2 & 0.0 & -0.2 & 0.2 & -0.1 & 0.7 & -0.3 \\
\hline 32 & $1.31 \cdot 10^{-3}$ & 0.48 & 1.216 & 1.8 & 1.7 & 0.8 & -0.1 & -0.6 & -0.3 & 0.1 & -0.3 & -0.3 & -0.7 & 0.8 & -0.4 \\
\hline 32 & $1.13 \cdot 10^{-3}$ & 0.56 & 1.249 & 2.0 & 1.8 & 1.0 & -0.3 & 0.8 & -0.7 & -0.5 & 0.2 & 0.3 & -0.5 & 0.4 & -0.4 \\
\hline 32 & $1.00 \cdot 10^{-3}$ & 0.63 & 1.252 & 2.6 & 2.3 & 1.5 & -0.7 & -0.3 & -1.1 & -0.5 & 0.4 & -0.3 & 0.9 & -0.1 & -0.4 \\
\hline 32 & $9.15 \cdot 10^{-4}$ & 0.69 & 1.387 & 2.8 & 3.5 & 1.7 & -0.9 & -0.1 & -2.1 & -0.7 & 0.5 & -0.2 & 1.1 & 1.3 & -0.5 \\
\hline 32 & $8.41 \cdot 10^{-4}$ & 0.75 & 1.291 & 3.3 & 4.0 & 2.3 & -1.7 & 0.3 & -2.6 & 0.6 & -0.3 & -0.3 & -0.3 & -0.4 & -0.6 \\
\hline 45 & $6.83 \cdot 10^{-3}$ & 0.13 & 0.803 & 1.2 & 1.7 & 0.5 & 0.0 & 0.8 & -0.7 & 0.0 & 0.2 & 0.1 & 0.5 & 1.1 & -0.1 \\
\hline 45 & $4.03 \cdot 10^{-3}$ & 0.22 & 0.942 & 1.3 & 2.1 & 0.6 & 0.0 & 1.5 & -0.1 & 0.1 & -0.5 & -0.2 & 0.4 & 1.2 & -0.2 \\
\hline 45 & $2.86 \cdot 10^{-3}$ & 0.31 & 1.057 & 1.5 & 1.1 & 0.7 & 0.0 & -0.1 & -0.1 & 0.1 & -0.4 & -0.1 & 0.1 & 0.7 & -0.3 \\
\hline 45 & $2.22 \cdot 10^{-3}$ & 0.40 & 1.107 & 1.7 & 1.5 & 0.8 & -0.1 & -0.4 & -0.2 & 0.0 & -0.3 & -0.3 & -0.7 & 0.7 & -0.3 \\
\hline 45 & $1.85 \cdot 10^{-3}$ & 0.48 & 1.105 & 2.0 & 1.4 & 1.0 & -0.2 & -0.4 & -0.4 & 0.2 & -0.3 & -0.1 & 0.4 & 0.5 & -0.4 \\
\hline 45 & $1.58 \cdot 10^{-3}$ & 0.56 & 1.260 & 2.2 & 2.3 & 1.2 & -0.3 & 0.1 & -0.5 & 1.0 & -0.6 & 0.2 & -1.0 & -0.8 & -0.4 \\
\hline 45 & $1.41 \cdot 10^{-3}$ & 0.63 & 1.293 & 2.8 & 2.4 & 1.4 & -0.3 & -0.7 & -1.0 & -0.9 & 0.7 & 0.3 & -0.8 & 0.4 & -0.4 \\
\hline 45 & $1.29 \cdot 10^{-3}$ & 0.69 & 1.227 & 3.2 & 3.0 & 1.9 & -0.8 & 0.6 & -1.5 & -0.3 & 0.7 & 0.1 & -0.4 & 0.9 & -0.5 \\
\hline 45 & $1.18 \cdot 10^{-3}$ & 0.75 & 1.228 & 3.7 & 4.5 & 2.4 & -1.4 & -2.1 & -2.0 & -0.5 & -0.3 & 0.6 & -1.0 & -1.2 & -0.6 \\
\hline 60 & $9.10 \cdot 10^{-3}$ & 0.13 & 0.746 & 1.3 & 1.9 & 0.6 & 0.0 & 1.0 & -0.7 & 0.1 & -0.6 & 0.3 & 0.5 & 1.0 & -0.1 \\
\hline 60 & $5.38 \cdot 10^{-3}$ & 0.22 & 0.868 & 1.5 & 2.6 & 0.7 & 0.0 & 1.4 & -0.2 & 0.0 & -0.3 & 0.3 & 1.2 & 1.7 & -0.2 \\
\hline 60 & $3.82 \cdot 10^{-3}$ & 0.31 & 0.994 & 1.8 & 1.1 & 0.8 & 0.0 & 0.2 & -0.1 & 0.1 & 0.1 & 0.4 & 0.3 & 0.5 & -0.3 \\
\hline 60 & $2.96 \cdot 10^{-3}$ & 0.40 & 1.068 & 2.1 & 1.5 & 0.9 & 0.0 & -0.8 & -0.2 & -0.1 & -0.1 & -0.1 & 0.3 & 0.6 & -0.3 \\
\hline 60 & $2.46 \cdot 10^{-3}$ & 0.48 & 1.112 & 2.4 & 1.6 & 1.2 & -0.2 & 0.3 & -0.2 & 0.3 & 0.6 & 0.2 & -0.2 & 0.5 & -0.3 \\
\hline 60 & $2.11 \cdot 10^{-3}$ & 0.56 & 1.126 & 2.7 & 2.3 & 1.3 & -0.3 & -0.2 & -0.5 & 0.6 & 1.1 & 0.1 & -0.3 & 1.1 & -0.4 \\
\hline 60 & $1.88 \cdot 10^{-3}$ & 0.63 & 1.215 & 3.4 & 3.4 & 1.8 & -0.4 & 1.5 & -0.8 & 0.3 & 1.8 & -0.2 & 0.6 & 1.0 & -0.4 \\
\hline 60 & $1.71 \cdot 10^{-3}$ & 0.69 & 1.290 & 3.7 & 3.2 & 2.2 & -0.8 & 0.9 & -1.6 & -0.4 & -0.3 & 0.5 & 0.7 & -0.7 & -0.5 \\
\hline 60 & $1.58 \cdot 10^{-3}$ & 0.75 & 1.221 & 4.2 & 3.7 & 2.4 & -0.9 & 1.0 & -1.9 & 0.7 & -1.0 & 0.3 & 0.4 & -1.0 & -0.4 \\
\hline
\end{tabular}

Continued on Next Page. . 


\begin{tabular}{|c|c|c|c|c|c|c|c|c|c|c|c|c|c|c|c|}
\hline $\begin{array}{r}Q^{2} \\
\left(\mathrm{GeV}^{2}\right)\end{array}$ & $x$ & $y$ & $\begin{array}{l}\tilde{\sigma}^{e^{+} p} \\
\text { LER }\end{array}$ & $\begin{array}{r}\delta_{\text {stat }} \\
(\%)\end{array}$ & $\begin{array}{r}\delta_{\text {sys }} \\
(\%)\end{array}$ & $\begin{array}{r}\delta_{\text {unc }} \\
(\%)\end{array}$ & $\begin{array}{l}\delta_{\gamma p} \\
(\%)\end{array}$ & $\begin{array}{l}\delta_{E_{e}} \\
(\%)\end{array}$ & $\begin{array}{l}\delta_{E_{h}} \\
(\%)\end{array}$ & $\begin{array}{r}\delta_{e I D} \\
(\%)\end{array}$ & $\begin{array}{l}\delta_{d x} \\
(\%)\end{array}$ & $\begin{array}{l}\delta_{d y} \\
(\%)\end{array}$ & $\begin{array}{r}\delta_{\mathrm{MVD}} \\
(\%)\end{array}$ & $\begin{array}{r}\delta_{\mathrm{CTD}} \\
(\%)\end{array}$ & $\begin{array}{r}\delta_{\text {diff }} \\
(\%)\end{array}$ \\
\hline 80 & $1.21 \cdot 10^{-2}$ & 0.13 & 0.708 & 1.6 & 1.6 & 0.7 & 0.0 & 0.9 & -0.6 & 0.0 & -0.4 & -0.2 & -0.6 & 0.4 & -0.1 \\
\hline 80 & $7.17 \cdot 10^{-3}$ & 0.22 & 0.882 & 1.7 & 1.8 & 0.8 & 0.0 & 1.5 & -0.2 & 0.1 & -0.3 & -0.2 & -0.4 & -0.2 & -0.1 \\
\hline 80 & $5.09 \cdot 10^{-3}$ & 0.31 & 0.954 & 2.0 & 1.1 & 0.9 & 0.0 & -0.2 & -0.1 & 0.1 & -0.2 & -0.4 & -0.3 & 0.3 & -0.2 \\
\hline 80 & $3.94 \cdot 10^{-3}$ & 0.40 & 1.020 & 2.4 & 2.2 & 1.1 & 0.0 & -1.1 & -0.1 & 0.1 & -0.2 & -0.5 & -1.0 & -1.0 & -0.3 \\
\hline 80 & $3.29 \cdot 10^{-3}$ & 0.48 & 1.076 & 2.8 & 1.9 & 1.4 & -0.2 & -0.6 & -0.1 & 0.1 & 0.5 & -0.5 & 0.3 & 0.6 & -0.4 \\
\hline 80 & $2.82 \cdot 10^{-3}$ & 0.56 & 1.101 & 3.1 & 3.8 & 1.6 & -0.3 & -1.1 & -0.4 & 1.0 & 0.2 & 0.4 & 2.3 & 2.0 & -0.3 \\
\hline 80 & $2.50 \cdot 10^{-3}$ & 0.63 & 1.113 & 4.0 & 3.2 & 2.0 & -0.3 & -0.6 & -0.7 & 0.6 & -1.0 & -0.3 & -0.5 & -1.7 & -0.4 \\
\hline 80 & $2.29 \cdot 10^{-3}$ & 0.69 & 1.074 & 4.8 & 5.2 & 3.4 & -1.4 & 1.6 & -1.7 & 1.0 & -1.1 & -0.5 & 1.0 & 2.1 & -0.4 \\
\hline 80 & $2.10 \cdot 10^{-3}$ & 0.75 & 1.241 & 4.8 & 4.9 & 2.9 & -0.9 & 1.9 & -1.1 & -1.6 & -1.5 & -1.3 & -0.3 & -1.8 & -0.5 \\
\hline 110 & $1.67 \cdot 10^{-2}$ & 0.13 & 0.666 & 1.8 & 1.4 & 0.8 & 0.0 & 0.7 & -0.7 & 0.1 & -0.4 & 0.1 & -0.4 & -0.3 & -0.1 \\
\hline 110 & $9.86 \cdot 10^{-3}$ & 0.22 & 0.812 & 2.0 & 1.9 & 0.9 & 0.0 & 1.4 & -0.1 & -0.2 & 0.1 & -0.5 & -0.4 & 0.2 & -0.2 \\
\hline 110 & $7.00 \cdot 10^{-3}$ & 0.31 & 0.853 & 2.4 & 1.8 & 1.1 & 0.0 & 0.2 & -0.1 & -0.1 & 0.6 & 0.3 & 0.9 & 0.9 & -0.2 \\
\hline 110 & $5.42 \cdot 10^{-3}$ & 0.40 & 0.964 & 2.9 & 2.4 & 1.2 & 0.0 & -1.4 & -0.1 & 0.1 & 0.8 & 0.6 & 1.0 & 0.4 & -0.3 \\
\hline 110 & $4.52 \cdot 10^{-3}$ & 0.48 & 1.053 & 3.2 & 1.9 & 1.5 & -0.1 & -0.5 & -0.2 & 0.5 & -0.6 & 0.1 & 0.5 & -0.4 & -0.3 \\
\hline 110 & $3.87 \cdot 10^{-3}$ & 0.56 & 1.026 & 3.8 & 3.1 & 1.7 & -0.2 & 0.4 & -0.3 & -1.8 & -0.9 & -0.2 & 0.6 & -1.2 & -0.4 \\
\hline 110 & $3.44 \cdot 10^{-3}$ & 0.63 & 1.086 & 4.9 & 3.9 & 2.8 & -0.7 & -0.9 & -0.6 & -0.4 & -1.2 & 0.4 & 1.7 & -0.9 & -0.5 \\
\hline 110 & $3.14 \cdot 10^{-3}$ & 0.69 & 1.141 & 5.4 & 4.3 & 3.2 & -0.7 & 0.5 & -0.8 & -0.1 & 1.3 & 0.5 & 1.9 & 1.2 & -0.4 \\
\hline 110 & $2.89 \cdot 10^{-3}$ & 0.75 & 0.916 & 9.4 & 7.6 & 5.2 & -1.0 & -1.8 & -1.4 & 1.5 & 1.7 & 3.0 & -1.1 & -2.8 & -0.4 \\
\hline
\end{tabular}

Table 3: The reduced cross section, $\tilde{\sigma}$, for the reaction $e^{+} p \rightarrow e^{+} X$ at $\sqrt{s}=225 \mathrm{GeV}$.

Further details as described in caption of Table 1. 


\begin{tabular}{|r|c||c|c||c|c|}
\hline$Q^{2}$ & $x$ & $F_{L}^{(1)}$ & $F_{L}^{(2)}$ & $F_{2}^{(1)}$ & $F_{2}^{(2)}$ \\
\hline $\left.\mathrm{GeV}^{2}\right)$ & & & & & \\
\hline 24 & $6.67 \cdot 10^{-4}$ & $0.29_{-0.11}^{+0.11}$ & $0.31_{-0.05}^{+0.16}$ & $1.403_{-0.025}^{+0.025}$ & $1.404_{-0.019}^{+0.031}$ \\
24 & $8.16 \cdot 10^{-4}$ & $0.25_{-0.13}^{+0.13}$ & $0.27_{-0.09}^{+0.17}$ & $1.340_{-0.029}^{+0.029}$ & $1.341_{-0.026}^{+0.033}$ \\
24 & $1.08 \cdot 10^{-3}$ & $0.46_{-0.22}^{+0.22}$ & $0.47_{-0.14}^{+0.30}$ & $1.260_{-0.028}^{+0.028}$ & $1.261_{-0.019}^{+0.036}$ \\
\hline 32 & $8.89 \cdot 10^{-4}$ & $0.18_{-0.11}^{+0.11}$ & $0.20_{-0.05}^{+0.16}$ & $1.397_{-0.023}^{+0.023}$ & $1.398_{-0.016}^{+0.031}$ \\
32 & $1.09 \cdot 10^{-3}$ & $0.26_{-0.13}^{+0.13}$ & $0.27_{-0.10}^{+0.15}$ & $1.324_{-0.021}^{+0.021}$ & $1.324_{-0.019}^{+0.023}$ \\
32 & $1.43 \cdot 10^{-3}$ & $0.27_{-0.19}^{+0.19}$ & $0.28_{-0.13}^{+0.23}$ & $1.229_{-0.023}^{+0.023}$ & $1.229_{-0.016}^{+0.028}$ \\
\hline 45 & $1.25 \cdot 10^{-3}$ & $0.14_{-0.11}^{+0.11}$ & $0.15_{-0.05}^{+0.15}$ & $1.324_{-0.024}^{+0.024}$ & $1.324_{-0.016}^{+0.032}$ \\
45 & $1.53 \cdot 10^{-3}$ & $-0.11_{-0.13}^{+0.13}$ & $0.00_{-0.00}^{+0.10}$ & $1.233_{-0.022}^{+0.022}$ & $1.246_{-0.009}^{+0.028}$ \\
45 & $2.02 \cdot 10^{-3}$ & $0.37_{-0.19}^{+0.19}$ & $0.38_{-0.12}^{+0.25}$ & $1.173_{-0.022}^{+0.022}$ & $1.173_{-0.015}^{+0.028}$ \\
\hline 60 & $1.67 \cdot 10^{-3}$ & $0.16_{-0.12}^{+0.12}$ & $0.17_{-0.07}^{+0.15}$ & $1.326_{-0.029}^{+0.029}$ & $1.326_{-0.019}^{+0.037}$ \\
60 & $2.04 \cdot 10^{-3}$ & $0.19_{-0.15}^{+0.15}$ & $0.21_{-0.10}^{+0.18}$ & $1.211_{-0.025}^{+0.025}$ & $1.211_{-0.017}^{+0.030}$ \\
60 & $2.69 \cdot 10^{-3}$ & $0.27_{-0.21}^{+0.21}$ & $0.28_{-0.14}^{+0.25}$ & $1.145_{-0.024}^{+0.024}$ & $1.145_{-0.014}^{+0.030}$ \\
\hline 80 & $2.22 \cdot 10^{-3}$ & $0.18_{-0.13}^{+0.13}$ & $0.19_{-0.09}^{+0.15}$ & $1.256_{-0.029}^{+0.029}$ & $1.256_{-0.022}^{+0.035}$ \\
80 & $2.72 \cdot 10^{-3}$ & $0.38_{-0.17}^{+0.17}$ & $0.39_{-0.13}^{+0.20}$ & $1.213_{-0.027}^{+0.027}$ & $1.214_{-0.021}^{+0.033}$ \\
80 & $3.59 \cdot 10^{-3}$ & $0.12_{-0.22}^{+0.22}$ & $0.13_{-0.12}^{+0.20}$ & $1.082_{-0.022}^{+0.022}$ & $1.081_{-0.010}^{+0.030}$ \\
\hline 110 & $3.06 \cdot 10^{-3}$ & $0.33_{-0.15}^{+0.15}$ & $0.34_{-0.11}^{+0.18}$ & $1.254_{-0.033}^{+0.033}$ & $1.255_{-0.028}^{+0.040}$ \\
110 & $3.74 \cdot 10^{-3}$ & $0.31_{-0.18}^{+0.18}$ & $0.33_{-0.15}^{+0.22}$ & $1.136_{-0.029}^{+0.029}$ & $1.138_{-0.022}^{+0.035}$ \\
110 & $4.93 \cdot 10^{-3}$ & $0.01_{-0.25}^{+0.25}$ & $0.03_{-0.03}^{+0.26}$ & $1.022_{-0.026}^{+0.026}$ & $1.022_{-0.006}^{+0.038}$ \\
\hline
\end{tabular}

Table 4: The measured values of $F_{L}$ and $F_{2}$ at 18 separate $\left(x, Q^{2}\right)$ points. The quoted uncertainties include both the statistical and systematic sources, whereas a $\pm 2.5 \%$ correlated normalisation uncertainty is not included on the $F_{L}$ and $F_{2}$ values. The (1) superscript indicates an unconstrained fit whereas the (2) superscript indicates that the constraints $F_{2} \geq 0$ and $0 \leq F_{L} \leq F_{2}$ were enforced by the prior.

\begin{tabular}{|r||c|c||c|c|}
\hline $\begin{array}{r}Q^{2} \\
\left(\mathrm{GeV}^{2}\right)\end{array}$ & $F_{L}^{(1)}$ & $F_{L}^{(2)}$ & $R^{(1)}$ & $R^{(2)}$ \\
\hline 24 & $0.30_{-0.09}^{+0.09}$ & $0.30_{-0.05}^{+0.12}$ & $0.27_{-0.07}^{+0.14}$ & $0.27_{-0.03}^{+0.19}$ \\
\hline 32 & $0.22_{-0.09}^{+0.09}$ & $0.22_{-0.05}^{+0.11}$ & $0.18_{-0.05}^{+0.12}$ & $0.18_{-0.03}^{+0.15}$ \\
\hline 45 & $0.10_{-0.08}^{+0.08}$ & $0.10_{-0.05}^{+0.10}$ & $0.08_{-0.05}^{+0.11}$ & $0.08_{-0.03}^{+0.13}$ \\
\hline 60 & $0.18_{-0.09}^{+0.09}$ & $0.18_{-0.06}^{+0.11}$ & $0.16_{-0.07}^{+0.13}$ & $0.16_{-0.05}^{+0.15}$ \\
\hline 80 & $0.24_{-0.10}^{+0.10}$ & $0.23_{-0.07}^{+0.13}$ & $0.23_{-0.09}^{+0.17}$ & $0.23_{-0.06}^{+0.20}$ \\
\hline 110 & $0.28_{-0.12}^{+0.12}$ & $0.28_{-0.10}^{+0.14}$ & $0.29_{-0.12}^{+0.23}$ & $0.29_{-0.10}^{+0.25}$ \\
\hline
\end{tabular}

Table 5: The single values of $F_{L}$ and $R$ extracted in each $Q^{2}$ bin. The quantities are quoted such that $Q^{2} / x$ was constant for each value, which corresponds to $y=0.71$ for $\sqrt{s}=225 \mathrm{GeV}$. The quoted uncertainties include both the statistical and systematic sources, although a $\pm 2.5 \%$ normalisation uncertainty is not included on the $F_{L}$ values. The (1) superscript indicates an unconstrained fit whereas the (2) superscript indicates that the constraints $F_{2} \geq 0$ and $0 \leq F_{L} \leq F_{2}$ were enforced in the prior. 


\section{ZEUS}
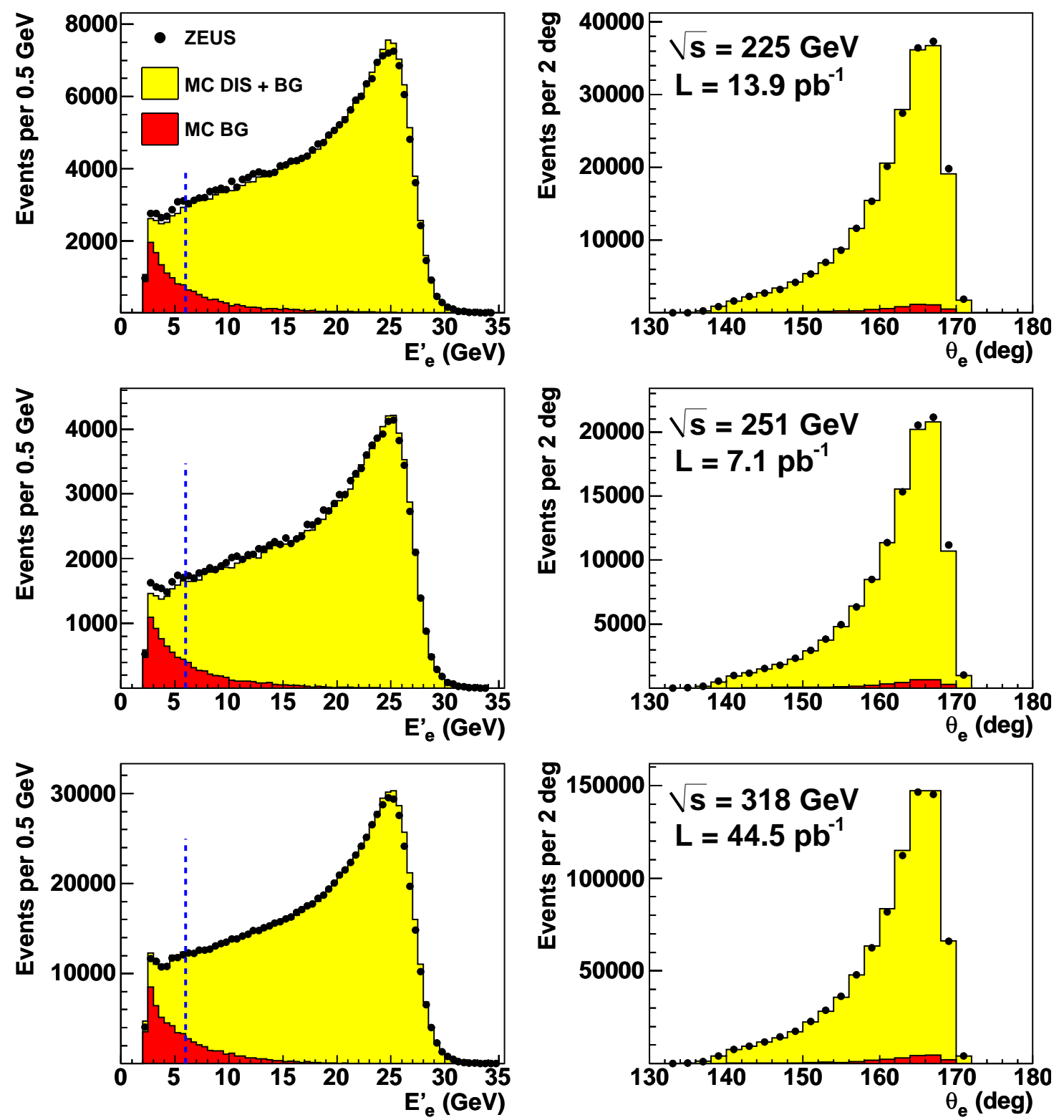

Figure 1: Detector-level distributions of the energy, $E_{e}^{\prime}$, and polar angle, $\theta_{e}$, of the scattered electron candidates within the HER, MER and LER data sets compared to the combined $M C$ predictions ( $M C D I S+B G$ ). The background only $M C$ is labelled $M C B G$. The vertical dashed-line represents the $E_{e}^{\prime}$ cut. The $\theta_{e}$ distributions are shown for $E_{e} \geq 6 \mathrm{GeV}$. 


\section{ZEUS}

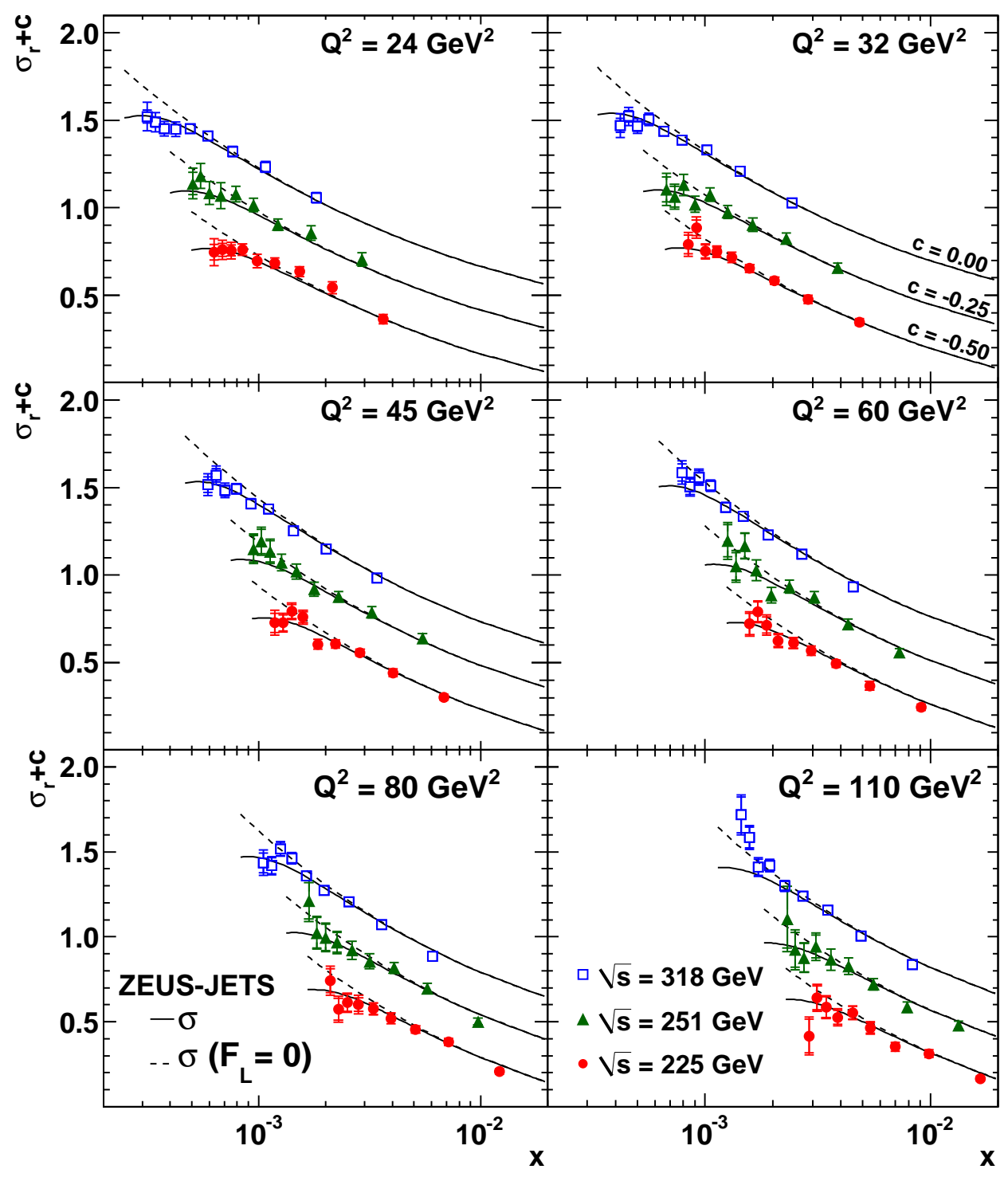

Figure 2: The reduced cross sections at 6 values of $Q^{2}$ as a function of $x$ for the three centre-of-mass energies. The points represent the ZEUS data from the HER $(\square), \operatorname{MER}(\mathbf{\Delta})$ and LER (•), respectively. The solid lines represent the predicted reduced cross sections, using the ZEUS-JETS PDFs. The dashed lines represent the predicted reduced cross sections when $F_{L}$ is set to zero. The points and lines are shifted by $c$ (see top right) for clarity. The inner error bars represent the statistical uncertainty. The outer error bars represent the statistical plus systematic uncertainties added in quadrature. A further $\pm 2.7 \%$ systematic normalisation uncertainty is not included. 


\section{ZEUS}

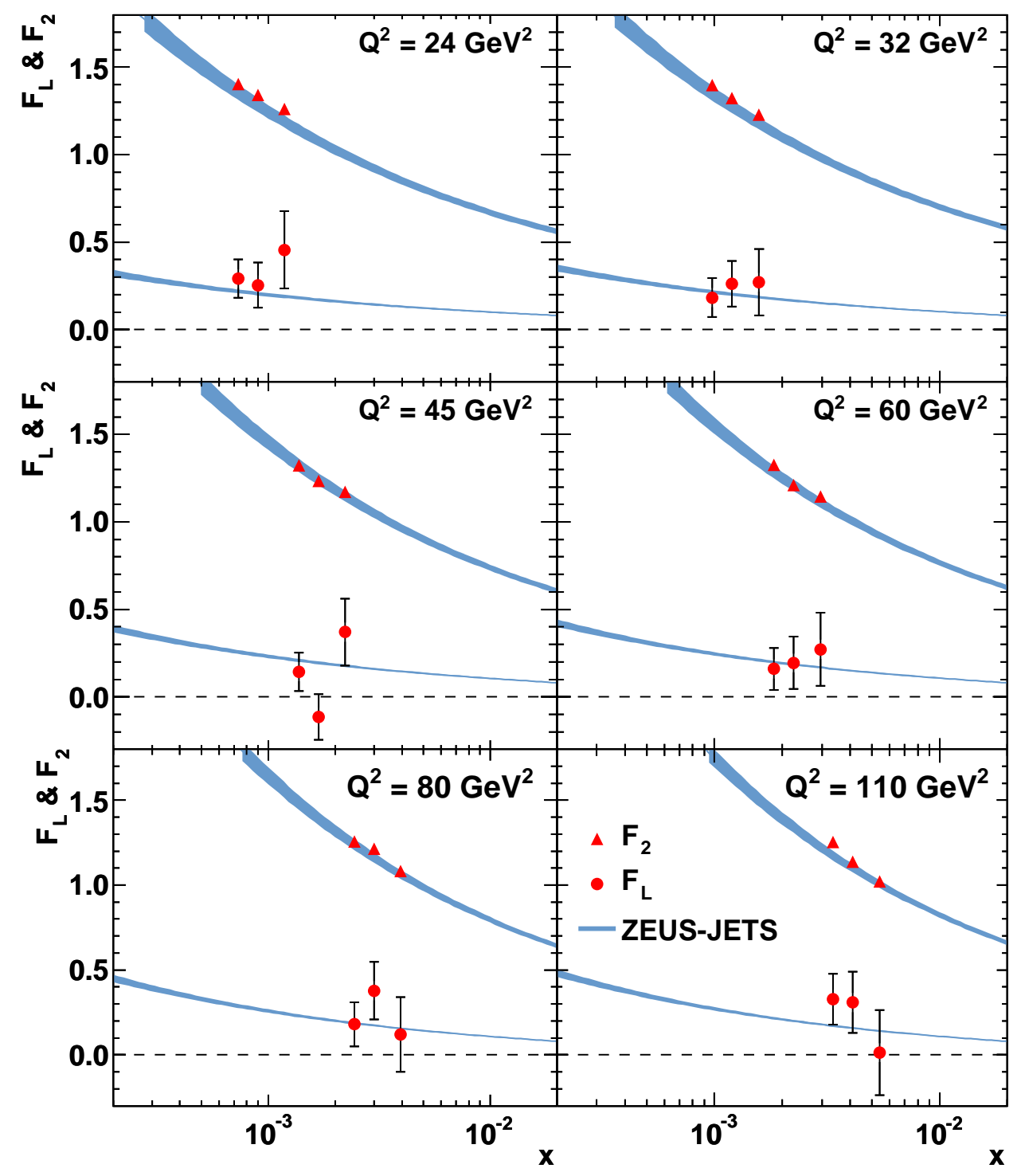

Figure 3: $F_{L}$ and $F_{2}$ at 6 values of $Q^{2}$ as a function of $x$. The points represent the ZEUS data for $F_{L}(\bullet)$ and $F_{2}(\mathbf{\Delta})$, respectively. The error bars on the data represent the combined statistical and systematic uncertainties. The error bars on $F_{2}$ are smaller than the symbols. A further $\pm 2.5 \%$ correlated normalisation uncertainty is not included. The DGLAP-predictions for $F_{L}$ and $F_{2}$ using the ZEUS-JETS PDFs are also shown. The bands indicate the uncertainty in the predictions. 

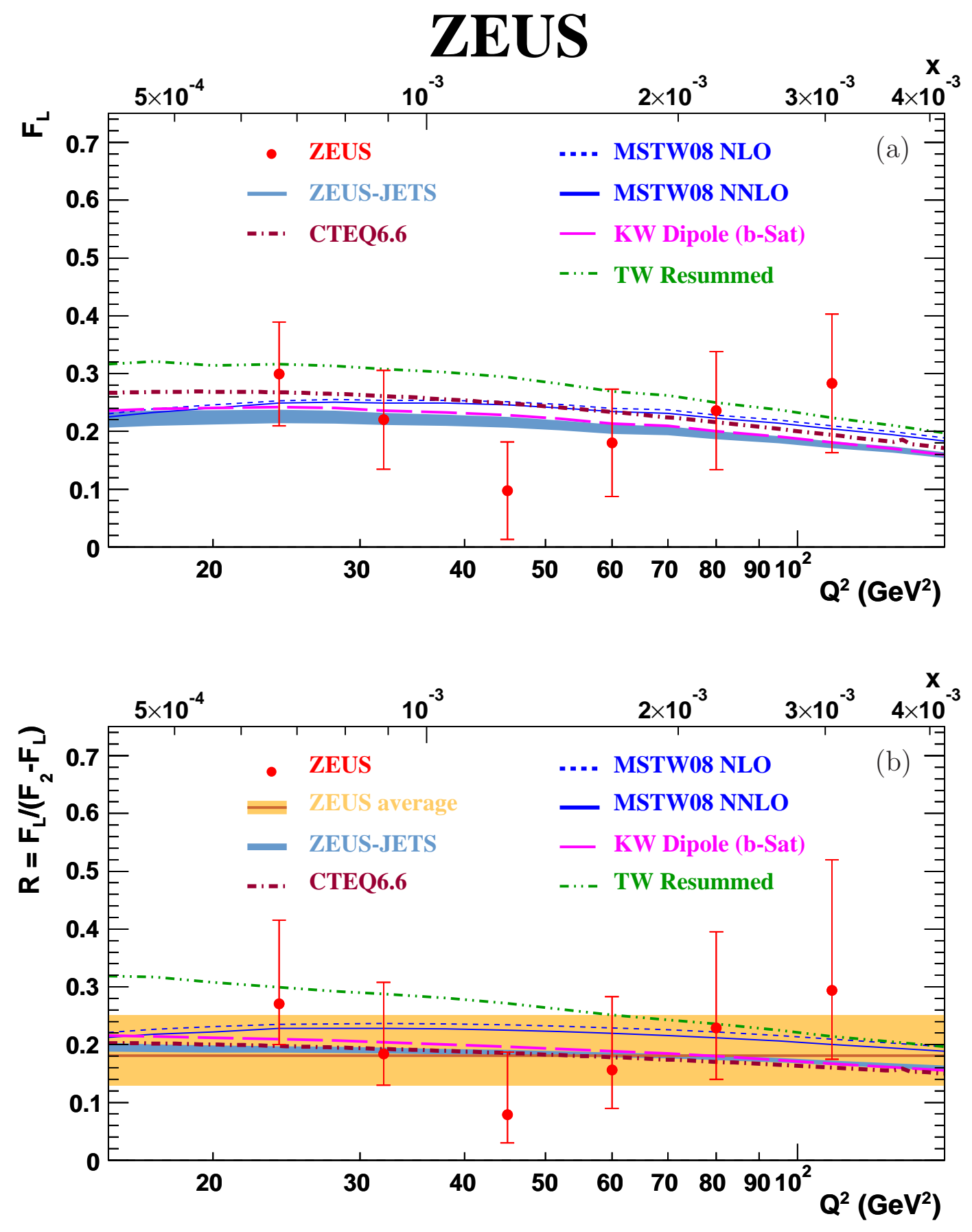

Figure 4: Values of (a) $F_{L}$ and (b) $R$ as a function of $Q^{2}$. The error bars on the data represent the combined statistical and systematic uncertainties. A further $\pm 2.5 \%$ correlated normalisation uncertainty is not included. The shaded band labelled ZEUS average represents the $68 \%$ probability interval for the overall $R$. The lines represent various model predictions (see text for details). 